\title{
Research Article \\ The Texture One-Zero Neutrino Mass Matrix with Vanishing Trace
}

\author{
Madan Singh \\ Department of Physics, National Institute of Technology Kurukshetra, Haryana 136119, India \\ Correspondence should be addressed to Madan Singh; singhmadan179@gmail.com
}

Received 14 October 2017; Accepted 6 December 2017; Published 5 April 2018

Academic Editor: Andrzej Okniński

Copyright ( 2018 Madan Singh. This is an open access article distributed under the Creative Commons Attribution License, which permits unrestricted use, distribution, and reproduction in any medium, provided the original work is properly cited. The publication of this article was funded by $\mathrm{SCOAP}^{3}$.

\begin{abstract}
In the light of latest neutrino oscillation data, we have investigated the one-zero Majorana neutrino mass matrix $M_{v}$, with zero sum condition of mass eigenvalues in the flavor basis, where charged lepton mass matrix is diagonal. Among the six possible one-zero cases, it is found that only five can survive the current experimental data, while case with $(1,1)$ vanishing element of $M_{\nu}$ is ruled out, if zero trace condition is imposed at $3 \sigma$ confidence level (CL). Numerical and some approximate analytical results are presented.
\end{abstract}

\section{Introduction}

The Double Chooz, Daya Bay, and RENO Collaborations [13] have finally established the nonzero and relatively large value of the reactor mixing angle $\theta_{13}$; hence the number of known available neutrino oscillation parameters approaches five, namely, two mass-squared differences $\left(\delta m^{2}, \Delta m^{2}\right)$ and three neutrino mixing angles $\left(\theta_{12}, \theta_{23}, \theta_{13}\right)$. However, any general $3 \times 3$ neutrino mass matrix contains more parameters than can be measured in realistic experiments. In fact, assuming the Majorana-type nature of neutrinos, the neutrino mass matrix contains nine real free parameters: three neutrino masses $\left(m_{1}, m_{2}, m_{3}\right)$, three flavor mixing angles $\left(\theta_{12}, \theta_{23}, \theta_{13}\right)$, and three CP violating phases $(\delta, \rho, \sigma)$.

In order to reduce the number of free parameters, several phenomenological ideas, in particular texture zeros [4-26], have been widely adopted in the literature. The imposition of texture zeros in neutrino mass matrix leads to some important phenomenological relations between flavor mixing angles and fermion mass ratios [24-27]. In the flavor basis, where charged lepton mass matrix is diagonal, at most two zeros are allowed in neutrino mass matrix, which are consistent with neutrino oscillation data $[25,26]$. The analysis of two texture zero neutrino mass matrices limits the number of experimentally viable cases to seven. The phenomenological implications of one texture zero neutrino mass matrix have also been studied in the literature [20-23] and it has been observed that all the six cases are viable with experimental data. However, the imposition of single texture zero condition in neutrino mass matrix makes larger parametric space for viability with the data available compared with two-zero texture. In order to impart predictability to one-zero texture, additional constraints in the form of vanishing determinant [28] or trace can be incorporated. The phenomenological implication of determinantless condition on one-zero texture have been rigorously studied in [20,28-32]. The implication of traceless condition was first put forward in [33] wherein the anomalies of solar and atmospheric neutrino oscillation experiments as well as the LSND experiment were simultaneously explained in the framework of three neutrinos. In [34], Zee has particularly investigated the case of $\mathrm{CP}$ conserving traceless neutrino mass matrix for explaining the solar and atmospheric neutrino deficits. Further motivation of traceless mass matrices can be provided by models wherein neutrino mass matrix can be constructed through a commutator of two matrices, as what happens in models of radiative mass generation [35]. In [36], Alhendi et al. have studied the case of two trackless submatrices of Majorana mass matrix in the flavor basis and carried out a detailed numerical analysis at $3 \sigma$ confidence level. The phenomenological implications of traceless neutrino mass matrix on neutrino masses, $\mathrm{CP}$ violating phases, and effective neutrino mass term are also 
studied in [37], for both normal and inverted mass ordering and in case of CP conservation and violation, respectively. In the present work we impose the traceless condition on texture one-zero Majorana mass matrix and investigate the outcomes of such condition on the parametric space of neutrino masses $\left(m_{1}, m_{2}, m_{3}\right)$ and CP violating phases $(\delta, \rho, \sigma)$.

Assuming the Majorana nature of neutrinos, neutrino mass matrix is complex symmetric. In the flavor basis, if one of the elements is considered to be zero, the number of possible cases turns out to be six, which are given below:

$$
\begin{aligned}
& T_{1}:\left(\begin{array}{ccc}
0 & \times & \times \\
\times & \times & \times \\
\times & \times & \times
\end{array}\right), \\
& T_{2}:\left(\begin{array}{ccc}
\times & \times & \times \\
\times & 0 & \times \\
\times & \times & \times
\end{array}\right), \\
& T_{3}:\left(\begin{array}{ccc}
\times & \times & \times \\
\times & \times & \times \\
\times & \times & 0
\end{array}\right) \text {; } \\
& T_{4}:\left(\begin{array}{ccc}
\times & 0 & \times \\
0 & \times & \times \\
\times & \times & \times
\end{array}\right), \\
& T_{5}:\left(\begin{array}{ccc}
\times & \times & 0 \\
\times & \times & \times \\
0 & \times & \times
\end{array}\right), \\
& T_{6}:\left(\begin{array}{ccc}
\times & \times & \times \\
\times & \times & 0 \\
\times & 0 & \times
\end{array}\right),
\end{aligned}
$$

where " $x$ " stands for nonzero element and complex matrix element.

Among these possible cases, there exists a permutation symmetry between certain pair of cases, namely, $\left(T_{2}, T_{3}\right)$ and $\left(T_{4}, T_{5}\right)$, while cases $T_{1}$ and $T_{6}$ transform onto themselves independently. The origin of permutation symmetry is explained from the fact that these pairs are related by exchange of 2-3 rows and 2-3 columns of neutrino mass matrix. The corresponding permutation matrix is given by

$$
P_{23}=\left(\begin{array}{lll}
1 & 0 & 0 \\
0 & 0 & 1 \\
0 & 1 & 0
\end{array}\right)
$$

which leads to the following relations among the neutrino oscillation parameters:

$$
\begin{aligned}
& \theta_{12}^{X}=\theta_{12}^{Y}, \\
& \theta_{23}^{X}=90^{\circ}-\theta_{23}^{Y},
\end{aligned}
$$

$$
\begin{aligned}
& \theta_{13}^{X}=\theta_{13}^{Y}, \\
& \delta^{X}=\delta^{Y} \pm 180^{\circ},
\end{aligned}
$$

where $X$ and $Y$ superscripts denote the cases related by 2-3 permutation symmetry.

The rest of the work is planned as follows: In Section 2, we discuss the methodology used to reconstruct the Majorana neutrino mass matrix and subsequently obtain some useful phenomenological relations of neutrino mass ratios and Majorana phases by incorporating texture one-zero and zero trace conditions simultaneously. In Section 3, we present the numerical analysis using some approximate analytical relations. In Section 4, we summarize our work.

\section{Formalism}

In the flavor basis, the Majorana neutrino mass matrix $M_{\gamma}$, depending on three neutrino masses $\left(m_{1}, m_{2}, m_{3}\right)$ and the flavor mixing matrix can be expressed as

$$
M_{v}=V\left(\begin{array}{ccc}
m_{1} & 0 & 0 \\
0 & m_{2} & 0 \\
0 & 0 & m_{3}
\end{array}\right) V^{T}
$$

The mixing matrix $V$ can be written as $V=U P$, where $U$ denotes the neutrino mixing matrix consisting of three flavor mixing angles and one Dirac-like CP violating phase, whereas the matrix $P$ is a diagonal phase matrix; that is, $P$ $=\operatorname{diag}\left(e^{i \rho}, e^{i \sigma}, 1\right)$ with $\rho$ and $\sigma$ being the two Majorana CP violating phases. The neutrino mass matrix $M_{v}$ can then be rewritten as

$$
M_{\nu}=\left(\begin{array}{ccc}
M_{e e} & M_{e \mu} & M_{e \tau} \\
M_{e \mu} & M_{\mu \mu} & M_{\mu \tau} \\
M_{e \tau} & M_{\mu \tau} & M_{\tau \tau}
\end{array}\right)=U\left(\begin{array}{ccc}
\lambda_{1} & 0 & 0 \\
0 & \lambda_{2} & 0 \\
0 & 0 & \lambda_{3}
\end{array}\right) U^{T}
$$

where $\lambda_{1}=m_{1} e^{2 i \rho}, \lambda_{2}=m_{2} e^{2 i \sigma}$, and $\lambda_{3}=m_{3}$.

For the purpose of calculations, we have adopted the parameterization of the mixing matrix $U$ considered by [5]; for example,

$U$

$$
=\left(\begin{array}{ccc}
c_{12} c_{13} & s_{12} c_{13} & s_{13} \\
-c_{12} s_{23} s_{13}-s_{12} c_{23} e^{-i \delta} & -s_{12} s_{23} s_{13}+c_{12} c_{23} e^{-i \delta} & s_{23} c_{13} \\
-c_{12} c_{23} s_{13}+s_{12} s_{23} e^{-i \delta} & -s_{12} c_{23} s_{13}-c_{12} s_{23} e^{-i \delta} & c_{23} c_{13}
\end{array}\right),
$$

where $c_{i j}=\cos \theta_{i j}, s_{i j}=\sin \theta_{i j}$ for $i, j=1,2,3$, and $\delta$ is the $\mathrm{CP}$ violating phase.

If one of the elements of $M_{v}$ is considered zero, that is, $M_{l m}=0$, it leads to the following constraint equation:

$$
U_{l 1} U_{m 1} \lambda_{1}+U_{l 2} U_{m 2} \lambda_{2}+U_{l 3} U_{m 3} \lambda_{3}=0,
$$

where $l, m$ run over $e, \mu$, and $\tau$. 
TABLE 1: Current neutrino oscillation parameters from global fits at $1 \sigma, 2 \sigma$, and $3 \sigma$ confidence level [39]. No (IO) refers to normal (inverted) neutrino mass ordering.

\begin{tabular}{lcccc}
\hline Parameter & Best fit & $1 \sigma$ & $2 \sigma$ & $3 \sigma$ \\
\hline$\delta m^{2}\left[10^{-5} \mathrm{eV}^{2}\right]$ & 7.60 & $7.42-7.79$ & $7.26-7.99$ & $7.11-8.18$ \\
$\left|\Delta m_{31}^{2}\right|\left[10^{-3} \mathrm{eV}^{2}\right](\mathrm{NO})$ & 2.48 & $2.41-2.53$ & $2.35-2.59$ & $2.30-2.65$ \\
$\left|\Delta m_{31}^{2}\right|\left[10^{-3} \mathrm{eV}^{2}\right](\mathrm{IO})$ & 2.38 & $2.32-2.43$ & $2.26-2.48$ & $2.20-2.54$ \\
$\theta_{12}$ & $34.6^{\circ}$ & $33.6^{\circ}-35.6^{\circ}$ & $32.7^{\circ}-36.7^{\circ}$ & $31.8^{\circ}-37.8^{\circ}$ \\
$\theta_{23}(\mathrm{NO})$ & $48.9^{\circ}$ & $41.7^{\circ}-50.7^{\circ}$ & $40.0^{\circ}-52.1^{\circ}$ & $38.8^{\circ}-53.3^{\circ}$ \\
$\theta_{23}(\mathrm{IO})$ & $49.2^{\circ}$ & $46.9^{\circ}-50.7^{\circ}$ & $41.3^{\circ}-52.0^{\circ}$ & $39.4^{\circ}-53.1^{\circ}$ \\
$\theta_{13}(\mathrm{NO})$ & $8.6^{\circ}$ & $8.4^{\circ}-8.9^{\circ}$ & $8.2^{\circ}-9.1^{\circ}$ & $7.9^{\circ}-9.3^{\circ}$ \\
$\theta_{13}(\mathrm{IO})$ & $8.7^{\circ}$ & $8.5^{\circ}-8.9^{\circ}$ & $8.2^{\circ}-9.1^{\circ}$ & $8.0^{\circ}-9.4^{\circ}$ \\
$\delta(\mathrm{NO})$ & $254^{\circ}$ & $182^{\circ}-353^{\circ}$ & $0^{\circ}-360^{\circ}$ & $0^{\circ}-360^{\circ}$ \\
$\delta(\mathrm{IO})$ & $266^{\circ}$ & $210^{\circ}-322^{\circ}$ & $0^{\circ}-16^{\circ} \oplus 155^{\circ}-360^{\circ}$ & $0^{\circ}-360^{\circ}$ \\
\hline
\end{tabular}

The traceless condition implies that sum of the mass eigenvalues in neutrino mass matrix is zero; that is,

$$
\lambda_{1}+\lambda_{2}+\lambda_{3}=0
$$

Using (7) and (8), we obtain

$$
\begin{aligned}
& \frac{\lambda_{1}}{\lambda_{3}}=\frac{U_{l 2} U_{m 2}-U_{l 3} U_{m 3}}{U_{l 1} U_{m 1}-U_{l 2} U_{m 2}}, \\
& \frac{\lambda_{2}}{\lambda_{3}}=\frac{U_{l 3} U_{m 3}-U_{l 1} U_{m 1}}{U_{l 1} U_{m 1}-U_{l 2} U_{m 2}} .
\end{aligned}
$$

The magnitudes of neutrino mass ratios are given by

$$
\begin{gathered}
\xi \equiv \frac{m_{1}}{m_{3}}=\left|\frac{U_{l 2} U_{m 2}-U_{l 3} U_{m 3}}{U_{l 1} U_{m 1}-U_{l 2} U_{m 2}}\right|, \\
\zeta \equiv \frac{m_{2}}{m_{3}}=\left|\frac{U_{l 3} U_{m 3}-U_{l 1} U_{m 1}}{U_{l 1} U_{m 1}-U_{l 2} U_{m 2}}\right| .
\end{gathered}
$$

Using (9), we find the following analytical relations for Majorana phases $(\rho, \sigma)$ :

$$
\begin{aligned}
& \rho=\frac{1}{2} \arg \left(\frac{U_{l 2} U_{m 2}-U_{l 3} U_{m 3}}{U_{l 1} U_{m 1}-U_{l 2} U_{m 2}}\right), \\
& \sigma=\frac{1}{2} \arg \left(\frac{U_{l 3} U_{m 3}-U_{l 1} U_{m 1}}{U_{l 1} U_{m 1}-U_{l 2} U_{m 2}}\right) .
\end{aligned}
$$

Thus neutrino mass ratios $(\xi, \zeta)$ and two Majorana-type CP violating phases $(\rho, \sigma)$ can fully be determined in terms of three mixing angles $\left(\theta_{12}, \theta_{23}, \theta_{13}\right)$ and the Dirac-type CP violating phase $(\delta)$. The ratio of two neutrino mass-squared differences in terms of neutrino mass ratios $\xi$ and $\zeta$ is given by

$$
R_{v}=\frac{\delta m^{2}}{\left|\Delta m^{2}\right|}=\frac{2\left(\zeta^{2}-\xi^{2}\right)}{\left|2-\left(\zeta^{2}+\xi^{2}\right)\right|},
$$

where $\delta m^{2}=\left(m_{2}^{2}-m_{1}^{2}\right)$ and $\Delta m^{2}=\left|m_{3}^{2}-(1 / 2)\left(m_{1}^{2}+m_{2}^{2}\right)\right|$ [38] corresponds to solar and atmospheric neutrino squared differences, respectively. The sign of $\Delta m^{2}$ is still not known experimentally; that is, $\Delta m^{2}>0$ or $\Delta m^{2}<0$ corresponds to the normal or inverted mass ordering of neutrinos.

The expressions for three neutrino masses $\left(m_{1}, m_{2}, m_{3}\right)$ can be given as

$$
\begin{aligned}
& m_{3}=\sqrt{\frac{\delta m^{2}}{\left(\zeta^{2}-\xi^{2}\right)}}, \\
& m_{2}=m_{3} \zeta, \\
& m_{1}=m_{3} \xi .
\end{aligned}
$$

Thus the neutrino mass spectrum can be fully determined.

The expression for Jarlskog rephasing parameter $J_{\mathrm{CP}}$, which is a measure of CP violation, is given by

$$
J_{\mathrm{CP}}=s_{12} c_{12} s_{23} c_{23} s_{13} c_{13}^{2} \sin \delta
$$

\section{Numerical Results and Discussion}

The experimental constraints on neutrino parameters at $1 \sigma$, $2 \sigma$, and $3 \sigma$ confidence level (CL) are given in Table 1 .

The effective Majorana mass term relevant for neutrinoless double beta $(0 \nu \beta \beta)$ decay is given by

$$
|M|_{e e}=\left|m_{1} c_{12}^{2} c_{13}^{2} e^{2 i \rho}+m_{2} s_{12}^{2} c_{13}^{2} e^{2 i \sigma}+m_{3} s_{13}^{2}\right| .
$$

The future observation of $0 \nu \beta \beta$ decay would imply lepton number violation and Majorana character of neutrinos. For recent reviews see [40-43]. There are a large number of projects such as CUORICINO [44], CUORE [45], GERDA [46], MAJORANA [47], SuperNEMO [48], EXO [49], and GENIUS [50] which target achieving a sensitivity up to $0.01 \mathrm{eV}$ for $|M|_{e e}$. For the present analysis, we assume the upper limit on $|M|_{e e}$ to be less than $0.5 \mathrm{eV}$ at $3 \sigma \mathrm{CL}$ [43]. The data collected from the Planck satellite [51] combined with other cosmological data put a limit on the sum of neutrino masses as

$$
\Sigma=\sum_{i=1}^{3} m_{i}<0.23 \mathrm{eV} \quad \text { at } 95 \% \mathrm{CL} .
$$

Here, we take rather more conservative limit on sum of neutrino masses $(\Sigma)$ (i.e., $\Sigma<1 \mathrm{eV}$ ) at $3 \sigma \mathrm{CL}$. We span 
TABLE 2: The exact expression of neutrino mass ratios $\xi$ and $\zeta$ of all the six one-zero textures with vanishing trace is shown. The symbols $c_{2(i j)} \equiv \cos 2 \theta_{i j}, s_{2(i j)} \equiv \sin 2 \theta_{i j}$ are defined.

\begin{tabular}{|c|c|}
\hline Cases & Analytical expressions for $\xi$ and $\zeta$ \\
\hline \multirow{2}{*}{$T_{1}$} & $\xi=+\sec 2 \theta_{12}\left(s_{12}^{2}-t_{13}^{2}\right)$ \\
\hline & $\zeta=-\sec 2 \theta_{12}\left(c_{12}^{2}-t_{13}^{2}\right)$ \\
\hline \multirow{4}{*}{$T_{2}$} & $\xi_{-}\left(s_{12}^{2} s_{13}^{2}-c_{13}^{2}\right) s_{23}^{2}+c_{12} c_{23}\left(c_{12} c_{23} e^{-i \delta}-2 s_{12} s_{23} s_{13}\right) e^{-i \delta}$ \\
\hline & $\zeta=\overline{\left(s_{23}^{2} s_{13}^{2}-c_{23}^{2} e^{-2 i \delta}\right) c_{2(12)}+s_{2(12)} s_{2(23)} s_{13} e^{-i \delta}}$ \\
\hline & $\zeta=\frac{\left(-c_{12}^{2} s_{13}^{2}+c_{13}^{2}\right) s_{23}^{2}-s_{12} c_{23}\left(s_{12} c_{23} e^{-i \delta}+2 c_{12} s_{23} s_{13}\right) e^{-i \delta}}{2}$ \\
\hline & $\left(s_{23}^{2} s_{13}^{2}-c_{23}^{2} e^{-2 i \delta}\right) c_{2(12)}+s_{2(12)} s_{2(23)} s_{13} e^{-i \delta}$ \\
\hline \multirow{4}{*}{$T_{3}$} & $\xi=\frac{\left(s_{12}^{2} s_{13}^{2}-c_{13}^{2}\right) c_{23}^{2}+c_{12} s_{23}\left(c_{12} s_{23} e^{-i \delta}+2 s_{12} c_{23} s_{13}\right) e^{-i \delta}}{2}$ \\
\hline & $\left(c_{23}^{2} s_{13}^{2}-s_{23}^{2} e^{-2 i \delta}\right) c_{2(12)}-s_{2(12)} s_{2(23)} s_{13} e^{-i \delta}$ \\
\hline & $\zeta=\frac{\left(-c_{12}^{2} s_{13}^{2}+c_{13}^{2}\right) c_{23}^{2}-s_{12} s_{23}\left(s_{12} s_{23} e^{-i \delta}-2 c_{12} c_{23} s_{13}\right) e^{-i \delta}}{}$ \\
\hline & $\left(c_{23}^{2} s_{13}^{2}-s_{23}^{2} e^{-2 i \delta}\right) c_{2(12)}-s_{2(12)} s_{2(23)} s_{13} e^{-i \delta}$ \\
\hline \multirow{4}{*}{$T_{4}$} & $\xi=-s_{12} c_{12} c_{23} e^{-i \delta}+s_{23} s_{13}\left(1+s_{12}^{2}\right)$ \\
\hline & $s_{23} s_{13}\left(c_{12}^{2}-s_{12}^{2}\right)+2 s_{12} c_{12} c_{23} e^{-i \delta}$ \\
\hline & $\zeta=-s_{12} c_{12} c_{23} e^{-i 0}-s_{23} s_{13}\left(1+c_{12}^{2}\right)$ \\
\hline & $s_{23} s_{13}\left(c_{12}^{2}-s_{12}^{2}\right)+2 s_{12} c_{12} c_{23} e^{-i \delta}$ \\
\hline \multirow{4}{*}{$T_{5}$} & $\xi=\frac{s_{12} c_{12} s_{23} e^{-i \delta}+c_{23} s_{13}\left(1+s_{12}^{2}\right)}{2}$ \\
\hline & $c_{23} s_{13}\left(c_{12}^{2}-s_{1 \delta}^{2}\right)-2 s_{12} c_{12} s_{23} e^{-i \delta}$ \\
\hline & $\zeta=\frac{s_{12} c_{12} s_{23} e^{-i \delta}-c_{23} s_{13}\left(1+c_{12}^{2}\right)}{2}$ \\
\hline & $\zeta-\frac{c_{23} s_{13}\left(c_{12}^{2}-s_{12}^{2}\right)-2 s_{12} c_{12} s_{23} e^{-i \delta}}{2}$ \\
\hline \multirow{4}{*}{$T_{6}$} & $\xi=\frac{s_{23} c_{23}\left(s_{12}^{2} s_{13}^{2}-c_{13}^{2}-c_{12}^{2} e^{-2 i \delta}\right)-c_{12} s_{12} s_{13}\left(c_{23}^{2}-s_{23}^{2}\right) e^{-i \delta}}{2}$ \\
\hline & $s_{23} c_{23}\left(s_{13}^{2}+e^{-2 i \delta}\right) c_{2(12)} e^{-i \delta}+2 s_{12} c_{12} s_{13} c_{2(23)} e^{-i \delta}$ \\
\hline & $\zeta=\frac{s_{23} c_{23}\left(-c_{12}^{2} s_{13}^{2}+c_{13}^{2}+s_{12}^{2} e^{-210}\right)-c_{12} s_{12} s_{13}\left(c_{23}^{2}-s_{23}^{2}\right) e^{-i 0}}{2}$ \\
\hline & $s_{23} c_{23}\left(s_{13}^{2}+e^{-2 i \delta}\right) c_{2(12)} e^{-i \delta}+2 s_{12} c_{12} s_{13} c_{2(23)} e^{-i \delta}$ \\
\hline
\end{tabular}

the parameter space of input neutrino oscillation parameters $\left(\theta_{12}, \theta_{23}, \theta_{13}, \delta m^{2}, \Delta m^{2}\right)$ by choosing the randomly generated points of the order of $10^{6-7}$. Using Eq. (12), the parameter space of CP violating phases $(\delta, \rho, \sigma)$, effective mass term $|M|_{e e}$, neutrino masses $\left(m_{1}, m_{2}, m_{3}\right)$ can be subsequently constrained. In order to interpret the phenomenological results, some approximate analytical relations (up to certain leading order term of $s_{13}$ ) have been used in the following discussion. The exact analytical relations of neutrino mass ratios $(\xi, \zeta)$ have been provided in Table 2.

3.1. Case $T_{1}$. Using (6) and (9), in the leading order term of $\theta_{13}$, we obtain the following analytical relations:

$$
\begin{aligned}
& \frac{\lambda_{1}}{\lambda_{3}} \approx \sec 2 \theta_{12} s_{12}^{2}, \\
& \frac{\lambda_{2}}{\lambda_{3}} \approx-\sec 2 \theta_{12} c_{12}^{2} .
\end{aligned}
$$

For NO, using (12), we obtain $R_{v} \approx \zeta^{2}-\xi^{2} \approx \sec 2 \theta_{12}$. Using $3 \sigma$ experimental range of oscillation parameters, we find $2.23 \leq$ $R_{v} \leq 4.02$, which excludes the experimental range of $R_{v}$ and for IO we have

$$
R_{\nu} \approx \frac{\sec 2 \theta_{12}}{\sec ^{2} 2 \theta_{12} c_{12}^{4}-1}
$$

which is again inconsistent with current experimental data as $R_{v}>0.75$. Therefore, Case $T_{1}$ is ruled out with the latest neutrino oscillation data at $3 \sigma \mathrm{CL}$.

3.2. Case $T_{2}$. Using (6) and (9), we obtain the following analytical relations in the leading order approximation of $\theta_{13}$ :

$$
\begin{aligned}
& \frac{\lambda_{1}}{\lambda_{3}} \approx-\sec 2 \theta_{12}\left(c_{12}^{2}-t_{23}^{2} e^{2 i \delta}\right), \\
& \frac{\lambda_{2}}{\lambda_{3}} \approx \sec 2 \theta_{12}\left(s_{12}^{2}-t_{23}^{2} e^{2 i \delta}\right) .
\end{aligned}
$$

From (19), one can obtain the neutrino mass ratios

$$
\begin{aligned}
& \xi \approx \sec 2 \theta_{12} \sqrt{c_{12}^{4}+t_{23}^{4}-2 c_{12}^{2} t_{23}^{2} \cos 2 \delta}, \\
& \zeta \approx \sec 2 \theta_{12} \sqrt{s_{12}^{4}+t_{23}^{4}-2 s_{12}^{2} t_{23}^{2} \cos 2 \delta}
\end{aligned}
$$

and the Majorana $\mathrm{CP}$ violating phases

$$
\begin{aligned}
& \rho \approx \frac{1}{2} \tan ^{-1}\left(-\frac{t_{23}^{2} \sin 2 \delta}{c_{12}^{2}-t_{23}^{2} \cos 2 \delta}\right)+O\left(s_{13}\right), \\
& \sigma \approx \frac{1}{2} \tan ^{-1}\left(-\frac{t_{23}^{2} \sin 2 \delta}{s_{12}^{2}-t_{23}^{2} \cos 2 \delta}\right)+O\left(s_{13}\right) .
\end{aligned}
$$

The correlation plots for case $T_{2}$ have been compiled in Figures 1(a), 1(b), 1(c), and 1(d) and Figures 2(a), 2(b), 2(c), 


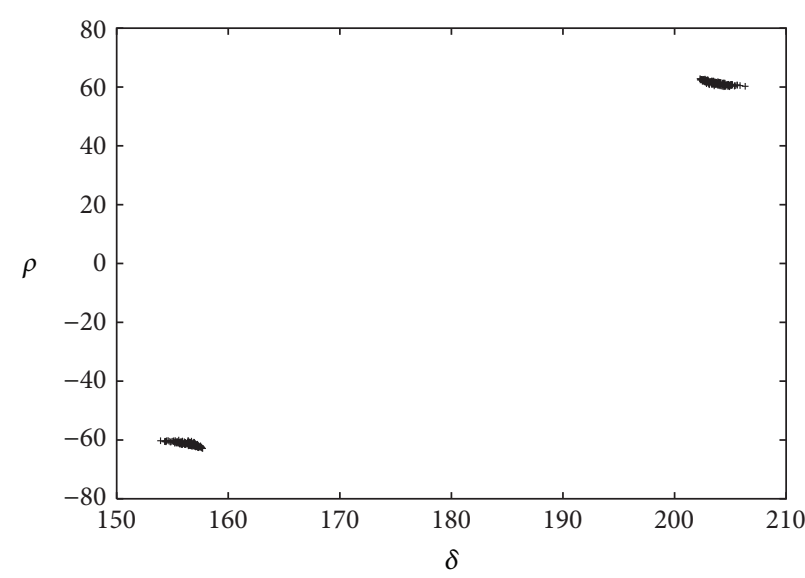

(a)

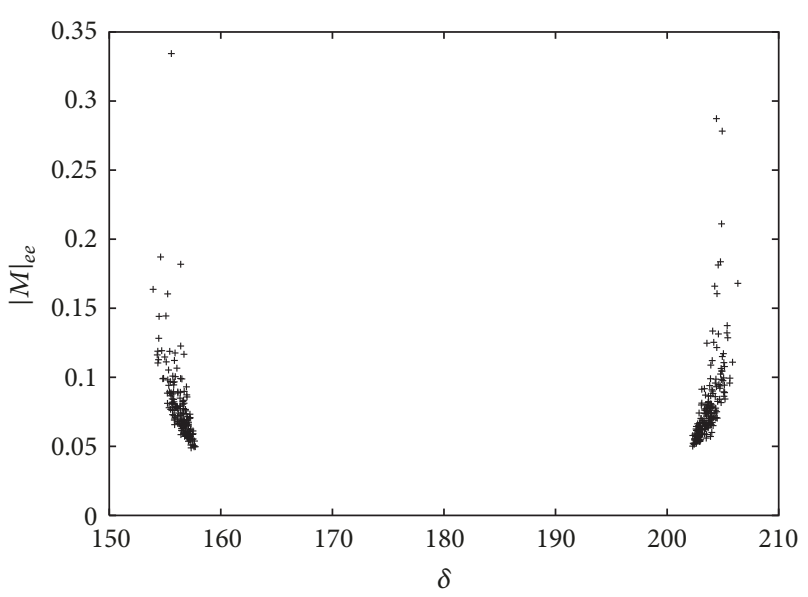

(c)

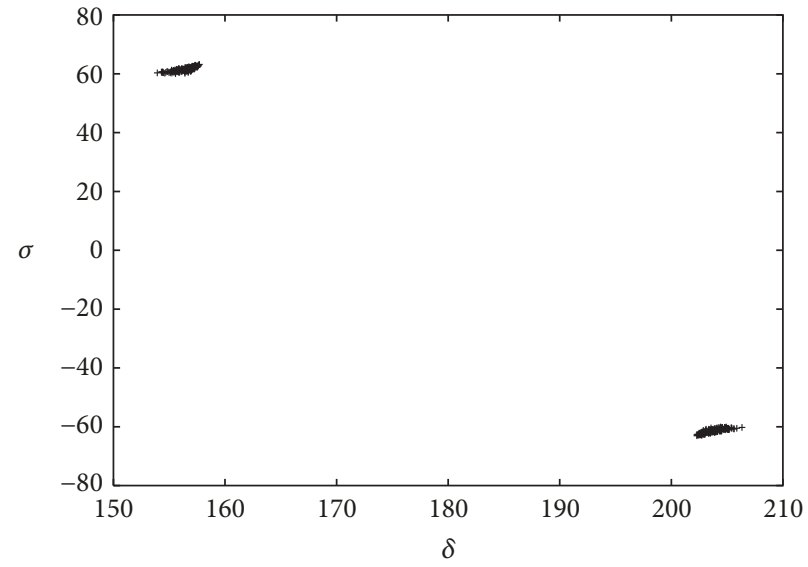

(b)

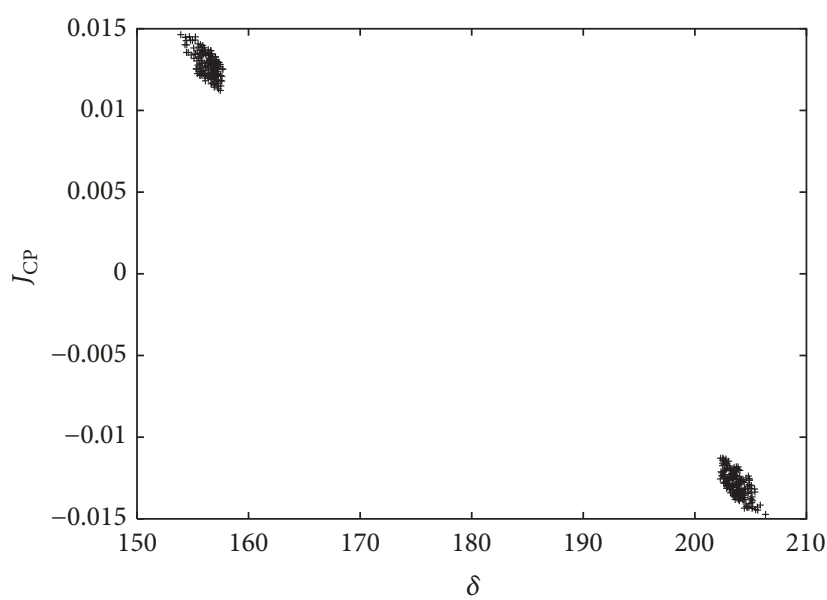

(d)

Figure 1: Case $T_{2}$ (NO): scattering plots of Majorana phases, Dirac CP violating phase $(\delta)$, effective neutrino mass $|M|_{e e}$, and Jarlskog rephrasing invariant $\left(J_{\mathrm{CP}}\right)$ have been shown. All the phase angles $(\delta, \rho, \sigma)$ are measured in degrees and $|M|_{e e}$ is in $\mathrm{eV}$ unit.

and 2(d), respectively. It is found from the analysis that case $\mathrm{T}_{2}$ favors both normal (NO) and inverted mass ordering (IO) at $3 \sigma \mathrm{CL}$. The parameter space of CP violating phases $\delta, \rho, \sigma$ is found to be constrained to very small ranges for $\mathrm{NO}$ (Figures 1 (a) and 1(b)). However, for IO, comparatively significant allowed parameter space is available for $\delta, \rho, \sigma$ (Figures 2(a) and $2(\mathrm{~b})$ ).

In the leading order approximation of $s_{13}$, the effective mass term in $0 \nu \beta \beta$ decay turns out to be

$$
|M|_{e e} \approx m_{3} t_{23}^{2} \approx 2.32 \times 10^{-2} \mathrm{eV}
$$

which lies well within the sensitivity limit of neutrinoless double beta decay experiments. Figures 1(c) and 2(c) show the correlation plot between $|M|_{e e}$ and $\delta$ for $\mathrm{NO}$ and IO, respectively. The Jarlskog rephrasing parameter $J_{\mathrm{CP}}$ is found to be nonvanishing for NO (Figure 1(d)); however, $J_{\mathrm{CP}}=0$ cannot be excluded for IO (Figure 2(d)).
3.3. Case $T_{3}$. With the help of (6) and (9), we deduce the following analytical expressions in the leading order of $s_{13}$ term.

$$
\begin{aligned}
& \frac{\lambda_{1}}{\lambda_{3}} \approx \sec 2 \theta_{12}\left(c_{12}^{2}-\frac{1}{t_{23}^{2}} e^{2 i \delta}\right), \\
& \frac{\lambda_{2}}{\lambda_{3}} \approx \sec 2 \theta_{12}\left(s_{12}^{2}-\frac{1}{t_{23}^{2}} e^{2 i \delta}\right) .
\end{aligned}
$$

From (23), one can obtain the neutrino mass ratios

$$
\begin{aligned}
& \xi \approx \sec 2 \theta_{12} \sqrt{c_{12}^{4}+\frac{1}{t_{23}^{4}}-2 c_{12}^{2} \frac{1}{t_{23}^{2}} \cos 2 \delta}, \\
& \zeta \approx \sec 2 \theta_{12} \sqrt{s_{12}^{4}+\frac{1}{t_{23}^{4}}-2 s_{12}^{2} \frac{1}{t_{23}^{2}} \cos 2 \delta}
\end{aligned}
$$




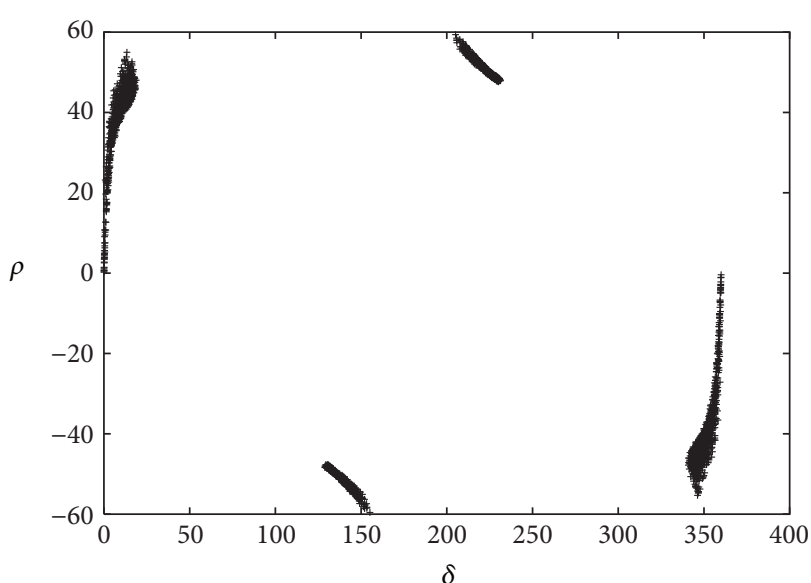

(a)

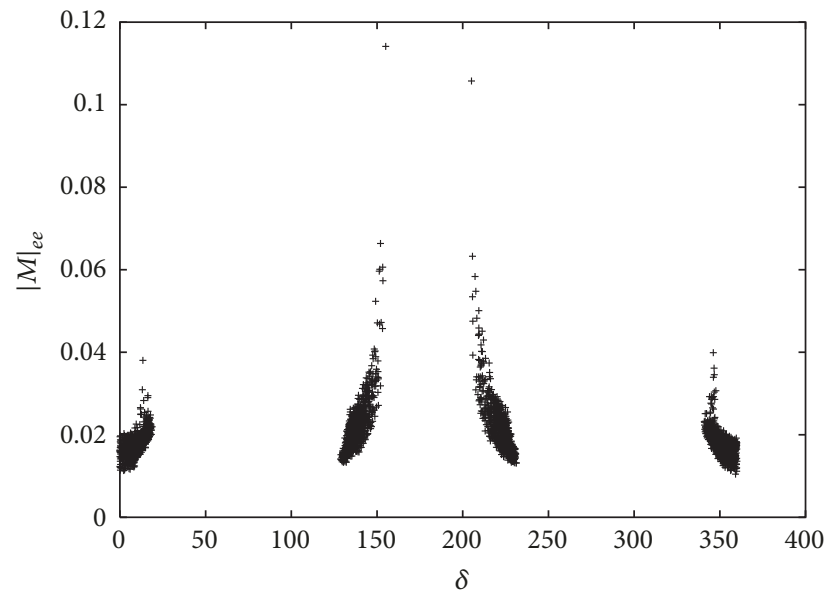

(c)

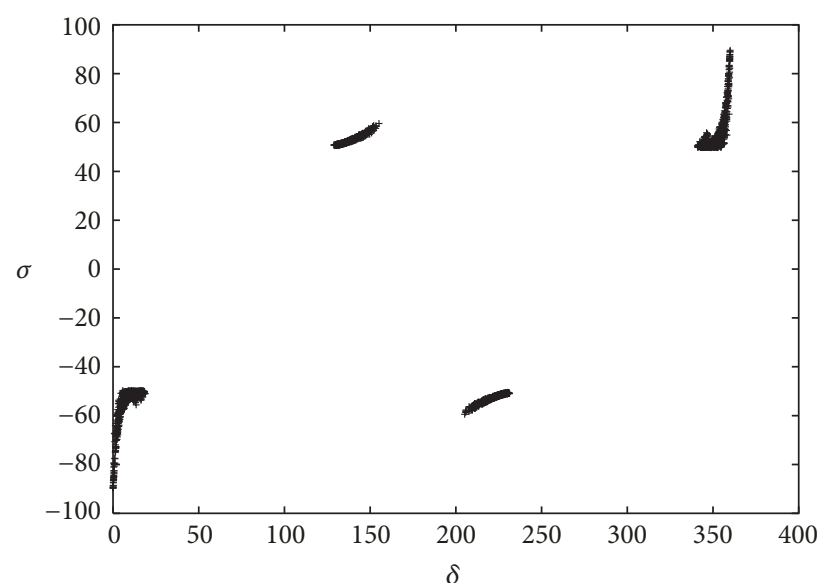

(b)

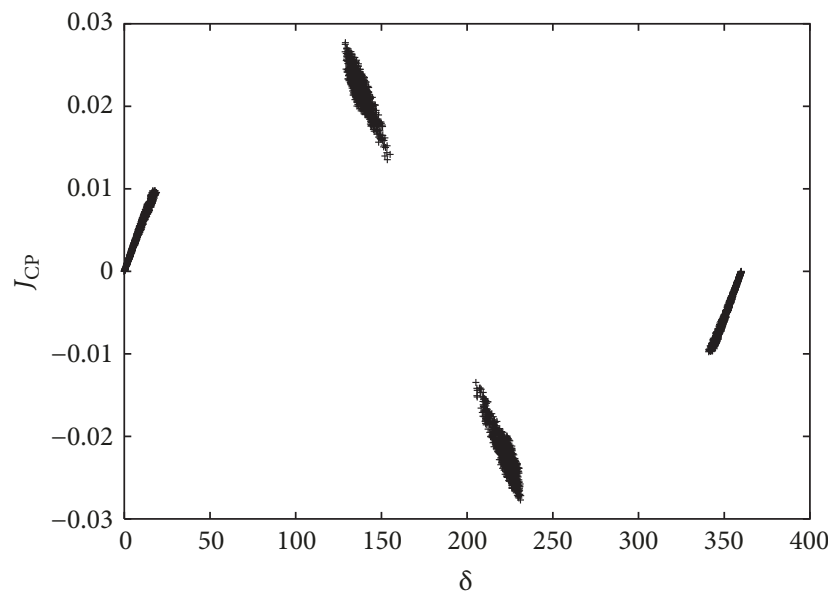

(d)

FIguRE 2: Case $T_{2}$ (IO): scattering plots of Majorana phases, Dirac CP violating phase $(\delta)$, effective neutrino mass $|M|_{e e}$, and Jarlskog rephrasing invariant $\left(J_{\mathrm{CP}}\right)$ have been shown. All the phase angles $(\delta, \rho, \sigma)$ are measured in degrees and $|M|_{e e}$ is in eV unit.

and the Majorana $\mathrm{CP}$ violating phases

$$
\begin{aligned}
& \rho \approx \frac{1}{2} \tan ^{-1}\left(-\frac{\sin 2 \delta}{c_{12}^{2} t_{23}^{2}-\cos 2 \delta}\right)+O\left(s_{13}\right), \\
& \sigma \approx \frac{1}{2} \tan ^{-1}\left(-\frac{\sin 2 \delta}{s_{12}^{2} t_{23}^{2}-t_{23}^{2} \cos 2 \delta}\right)+O\left(s_{13}\right) .
\end{aligned}
$$

Cases $T_{2}$ and $T_{3}$ are related via permutation symmetry; therefore the phenomenological results for case $T_{3}$ can be obtained from case $T_{3}$ by using (3). The correlation plots for case $T_{3}$ have been complied in Figures 3(a), 3(b), 3(c), and 3(d) (NO) and Figures 4(a), 4(b), 4(c), and 4(d) (IO), indicating the parameter space of $\rho, \sigma, \delta,|M|_{e e}, J_{\mathrm{CP}}$.

3.4. Case $T_{4}$. Using (6) and (9), we deduce the following analytical expressions in the leading order of $s_{13}$ term:

$$
\begin{aligned}
\frac{\lambda_{1}}{\lambda_{3}} & \approx \frac{\lambda_{2}}{\lambda_{3}} \approx-0.5, \\
\xi & \approx \zeta \approx 0.5 .
\end{aligned}
$$

Since $R_{v}=0$ in the leading order approximation of $s_{13}$, we have to work next to leading order, and we get

$$
\begin{aligned}
& \frac{\lambda_{1}}{\lambda_{3}} \approx-\frac{1}{2}\left(1-\frac{3}{2} \frac{s_{23} s_{13}}{c_{12} s_{12} c_{23}} e^{i \delta}\right)+O\left(s_{13}^{2}\right), \\
& \frac{\lambda_{2}}{\lambda_{3}} \approx-\frac{1}{2}\left(1+\frac{3}{2} \frac{s_{23} s_{13}}{c_{12} s_{12} c_{23}} e^{i \delta}\right)+O\left(s_{13}^{2}\right) .
\end{aligned}
$$

Using (27), the neutrino mass ratios can be given as

$$
\begin{aligned}
& \xi \approx \frac{1}{2} \sqrt{1+\frac{9}{4} \frac{s_{23}^{2} s_{13}^{2}}{c_{12}^{2} s_{12}^{2} c_{23}^{2}}-\frac{3 s_{23} s_{13}}{c_{12} s_{12} c_{23}} \cos \delta}, \\
& \zeta \approx \frac{1}{2} \sqrt{1+\frac{9}{4} \frac{s_{23}^{2} s_{13}^{2}}{c_{12}^{2} s_{12}^{2} c_{23}^{2}}+\frac{3 s_{23} s_{13}}{c_{12} s_{12} c_{23}} \cos \delta},
\end{aligned}
$$




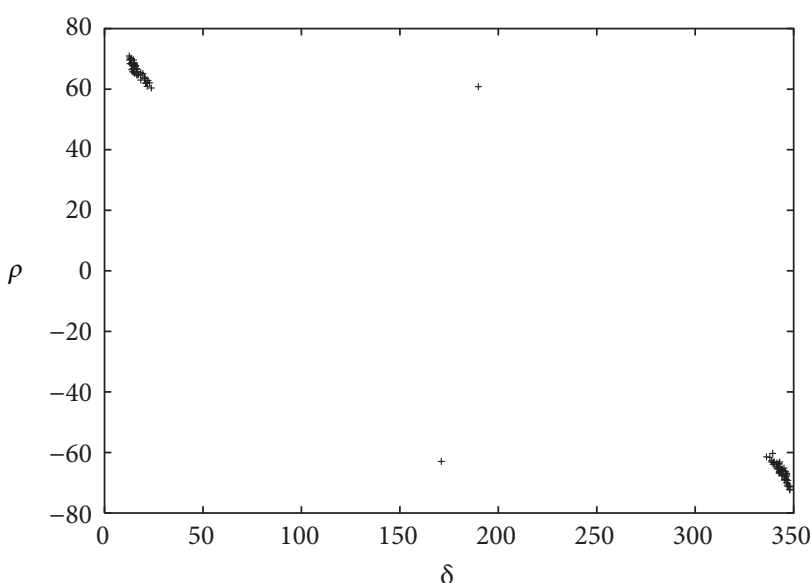

(a)

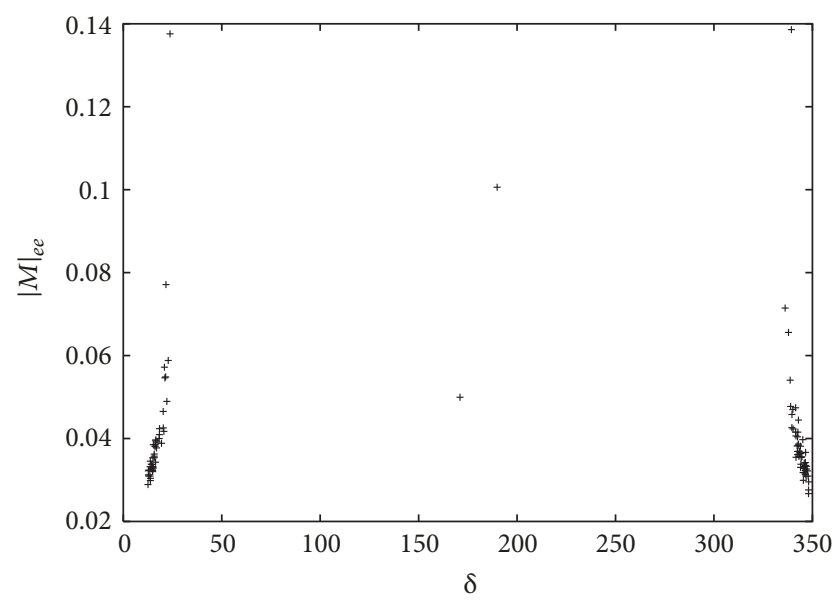

(c)

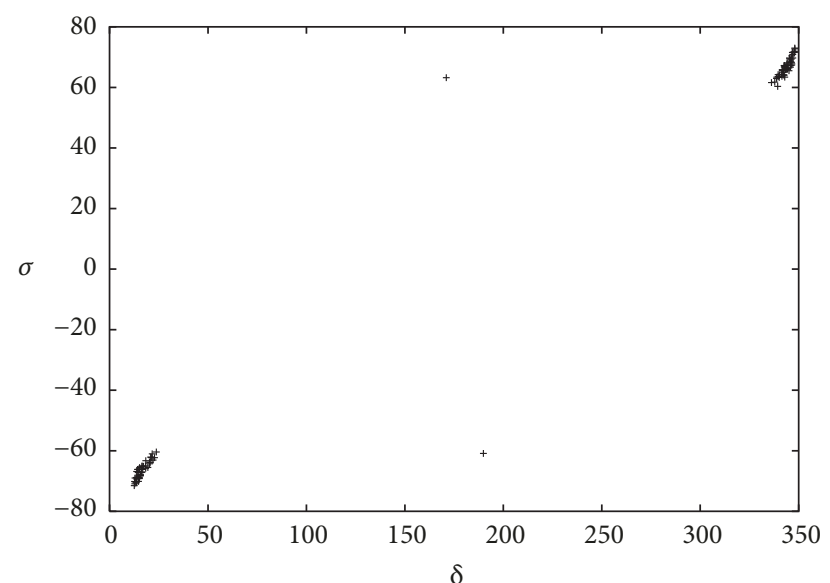

(b)

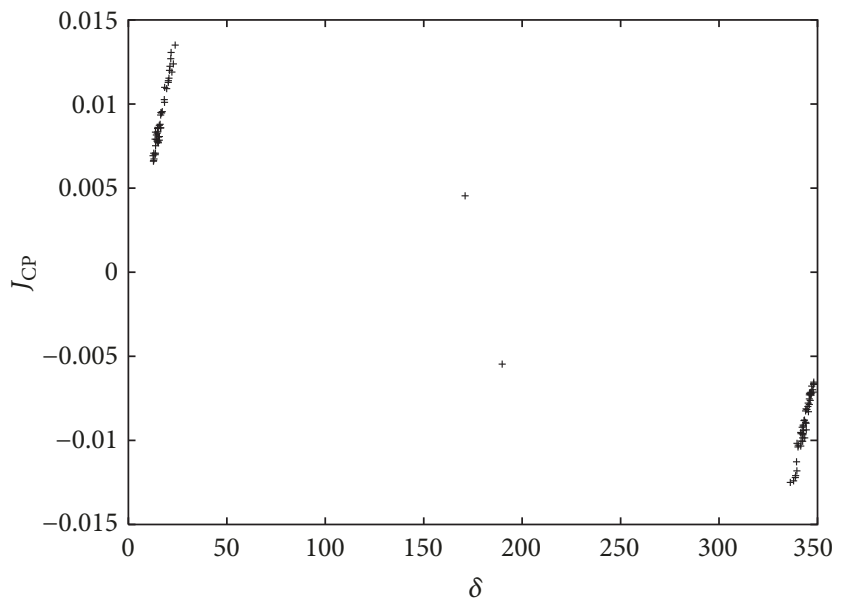

(d)

Figure 3: Case $T_{3}(\mathrm{NO})$ : scattering plots of Majorana phases, Dirac CP violating phase $(\delta)$, effective neutrino mass $|M|_{e e}$, and Jarlskog rephrasing invariant $\left(J_{\mathrm{CP}}\right)$ have been shown. All the phase angles $(\delta, \rho, \sigma)$ are measured in degrees and $|M|_{e e}$ is in $\mathrm{eV}$ unit.

and the Majorana CP violating phases are as

$$
\begin{aligned}
& \rho \approx \frac{1}{2} \tan ^{-1}\left(\frac{3 s_{23} s_{13} \sin \delta}{3 s_{23} s_{13} \cos \delta-2 c_{12} s_{12} c_{23}}\right)+O\left(s_{13}^{2}\right), \\
& \sigma \approx \frac{1}{2} \tan ^{-1}\left(\frac{3 s_{23} s_{13} \sin \delta}{3 s_{23} s_{13} \cos \delta+2 c_{12} s_{12} c_{23}}\right)+O\left(s_{13}^{2}\right) .
\end{aligned}
$$

Using the best fit values from latest global fits on neutrino oscillation data (Table 1), the neutrino mass spectrum can be given as follows:

$$
\begin{aligned}
& m_{3}=\sqrt{\frac{\delta m^{2}}{\left(\zeta^{2}-\xi^{2}\right)}} \approx 4.87 \times 10^{-2} \mathrm{eV} \\
& m_{2}=m_{3} \zeta \approx 2.85 \times 10^{-2} \mathrm{eV}, \\
& m_{1}=m_{3} \xi \approx 2.71 \times 10^{-2} \mathrm{eV},
\end{aligned}
$$

implying that only $\mathrm{NO}$ is allowed. Figures 5(a) and 5(b) show the correlation plot between Majorana phases $(\rho, \sigma)$ and Dirac CP violating phase $(\delta)$. The parameter space for $\delta$ is found to be restricted near $90^{\circ}$ and $270^{\circ}$. The prediction is significant considering the latest hint on $\delta$ near $270^{\circ}$ in the recent global fits on neutrino oscillation data (Table 1). The Majorana phases $(\rho, \sigma)$ are found to be constrained near $-90^{\circ}$ and $90^{\circ}$. In Figure 5(d), it is explicitly shown that $J_{\mathrm{CP}}$ is nonzero implying that case $T_{5}$ is necessarily $\mathrm{CP}$ violating.

In the leading order of $s_{13}$, the effective mass term in $0 \nu \beta \beta$ decay can be approximated as

$$
|M|_{e e} \approx \frac{m_{3}}{2} \approx 2.43 \times 10^{-2} \mathrm{eV},
$$

which is well within the accessible limit of next generation neutrinoless double decay experiments. The correlation plot between $|M|_{e e}$ and $\delta$ has been provided for case $T_{4}$ in Figure 5(c). 


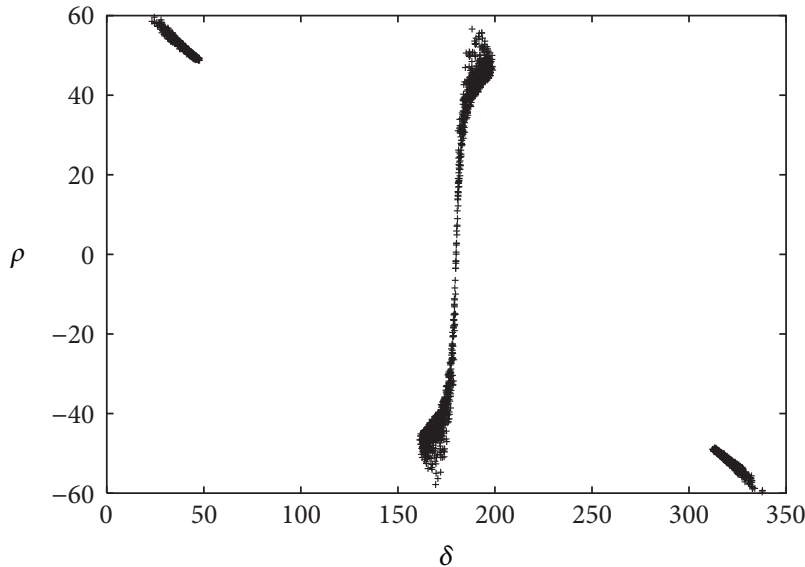

(a)

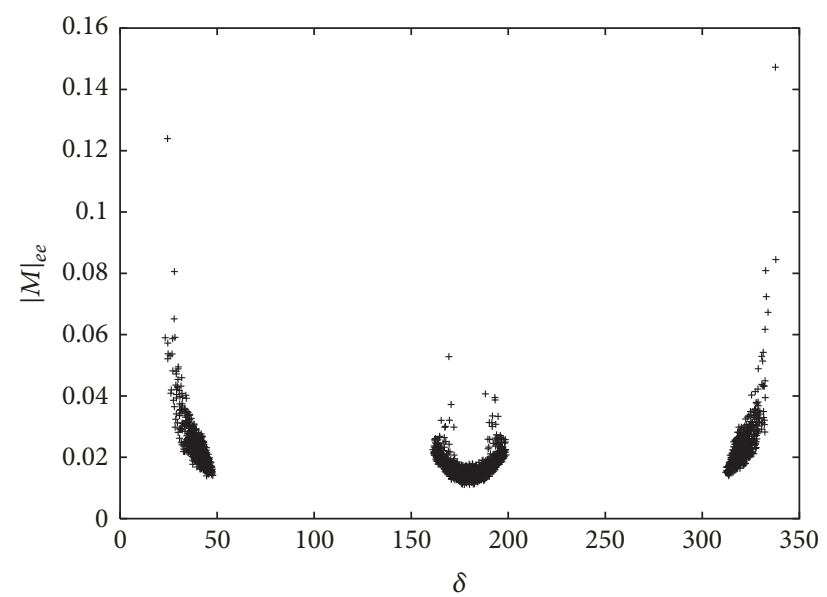

(c)

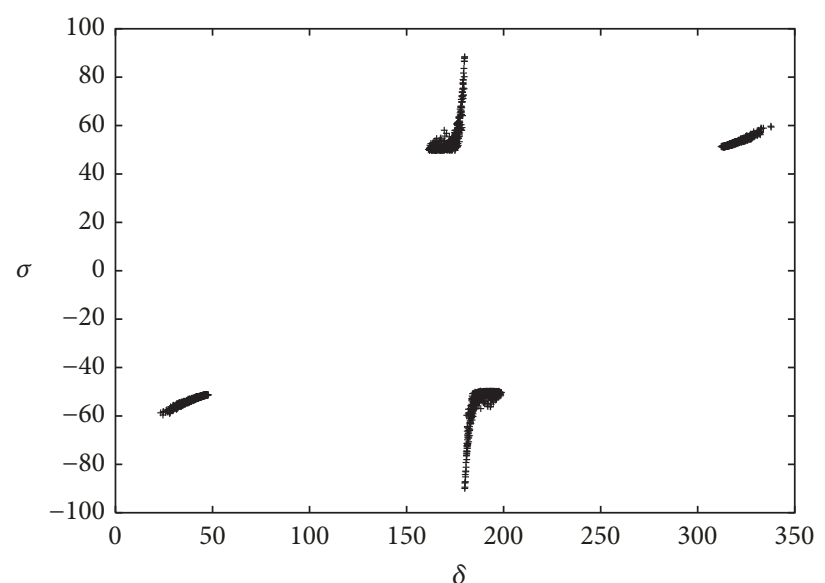

(b)

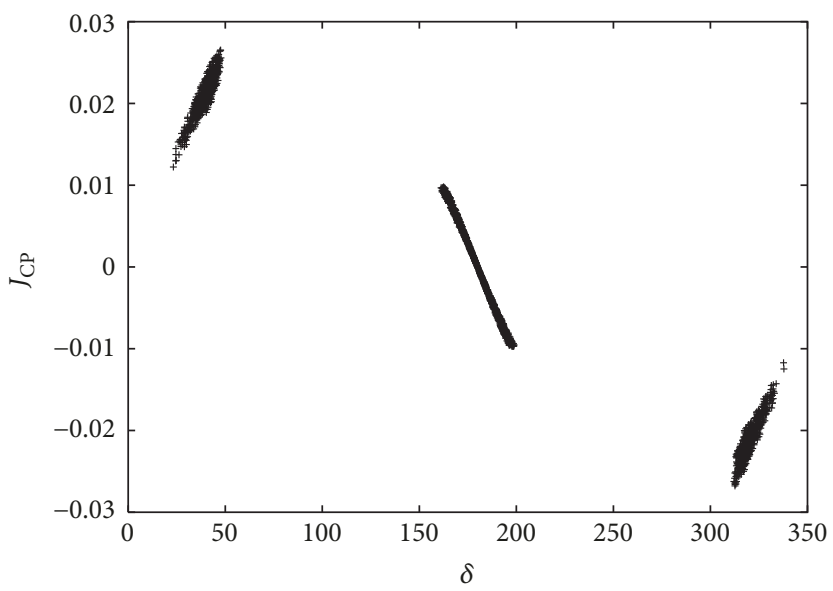

(d)

Figure 4: Case $T_{3}$ (IO): scattering plots of Majorana phases, Dirac CP violating phase $(\delta)$, effective neutrino mass $|M|_{e e}$, and Jarlskog rephrasing invariant $\left(J_{\mathrm{CP}}\right)$ have been shown. All the phase angles $(\delta, \rho, \sigma)$ are measured in degrees and $|M|_{e e}$ is in eV unit.

3.5. Case $T_{5}$. With the help of (6) and (9), we obtain the following analytical expressions in the leading order of $s_{13}$ term:

$$
\begin{aligned}
\frac{\lambda_{1}}{\lambda_{3}} & \approx \frac{\lambda_{2}}{\lambda_{3}} \approx 0.5, \\
\xi & \approx \zeta \approx 0.5 .
\end{aligned}
$$

Since $R_{v}=0$ in the leading order approximation of $s_{13}$, we have to work next to leading order, and we obtain

$$
\begin{aligned}
& \frac{\lambda_{1}}{\lambda_{3}} \approx-\frac{1}{2}\left(1+\frac{3}{2} \frac{c_{23} s_{13}}{c_{12} s_{12} s_{23}} e^{i \delta}\right)+O\left(s_{13}^{2}\right), \\
& \frac{\lambda_{2}}{\lambda_{3}} \approx-\frac{1}{2}\left(1-\frac{3}{2} \frac{c_{23} s_{13}}{c_{12} s_{12} s_{23}} e^{i \delta}\right)+O\left(s_{13}^{2}\right) .
\end{aligned}
$$

From (33), the neutrino mass ratios can be given as follows:

$$
\begin{gathered}
\xi \approx \frac{1}{2} \sqrt{1+\frac{9}{4} \frac{c_{23}^{2} s_{13}^{2}}{c_{12}^{2} s_{12}^{2} s_{23}^{2}}-\frac{3 c_{23} s_{13}}{c_{12} s_{12} s_{23}} \cos \delta}, \\
\zeta \approx \frac{1}{2} \sqrt{1+\frac{9}{4} \frac{c_{23}^{2} s_{13}^{2}}{c_{12}^{2} s_{12}^{2} s_{23}^{2}}+\frac{3 c_{23} s_{13}}{c_{12} s_{12} s_{23}} \cos \delta},
\end{gathered}
$$

and the Majorana CP violating phases are given as

$$
\begin{aligned}
& \rho \approx \frac{1}{2} \tan ^{-1}\left(\frac{3 c_{23} s_{13} \sin \delta}{3 c_{23} s_{13} \cos \delta+2 c_{12} s_{12} s_{23}}\right)+O\left(s_{13}^{2}\right), \\
& \sigma \approx \frac{1}{2} \tan ^{-1}\left(\frac{3 c_{23} s_{13} \sin \delta}{3 c_{23} s_{13} \cos \delta-2 c_{12} s_{12} s_{23}}\right)+O\left(s_{13}^{2}\right) .
\end{aligned}
$$




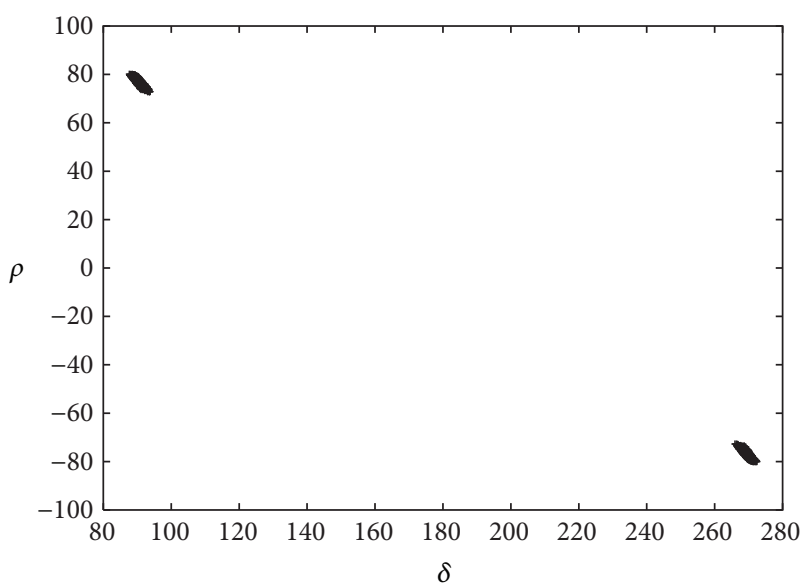

(a)

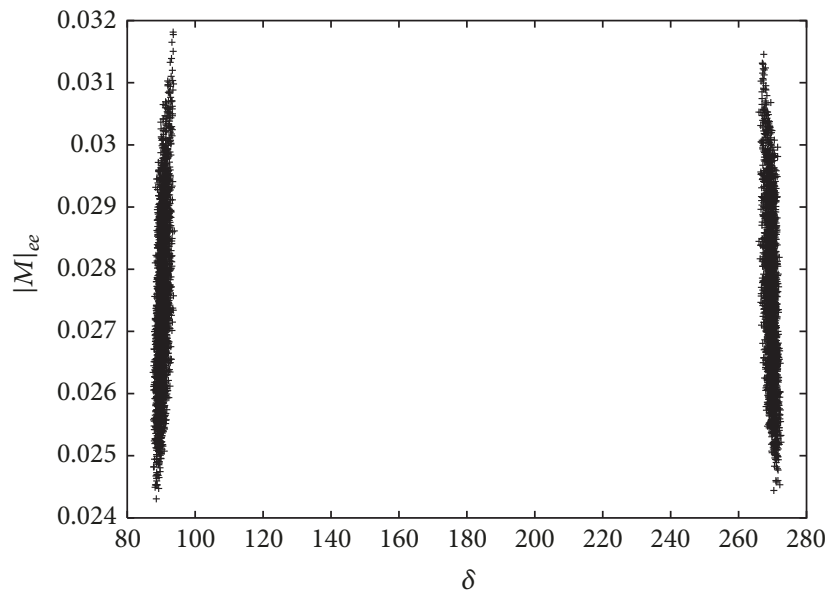

(c)

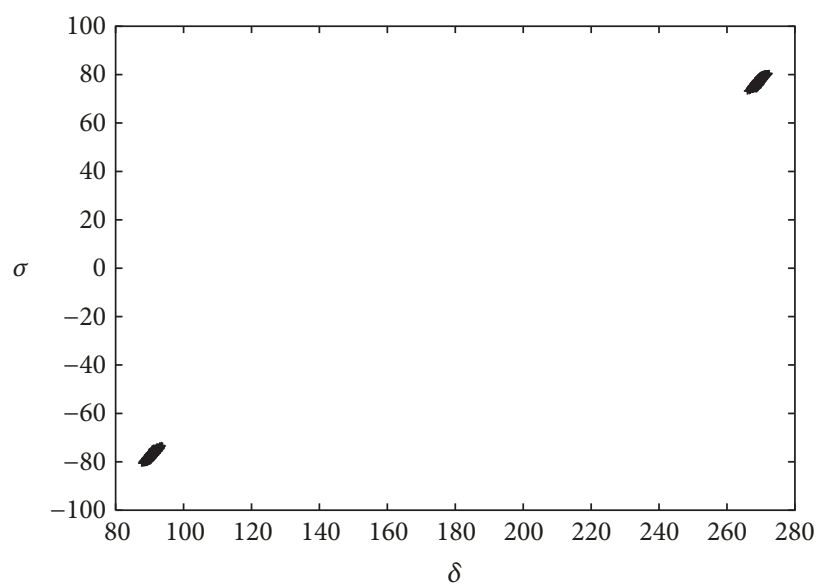

(b)

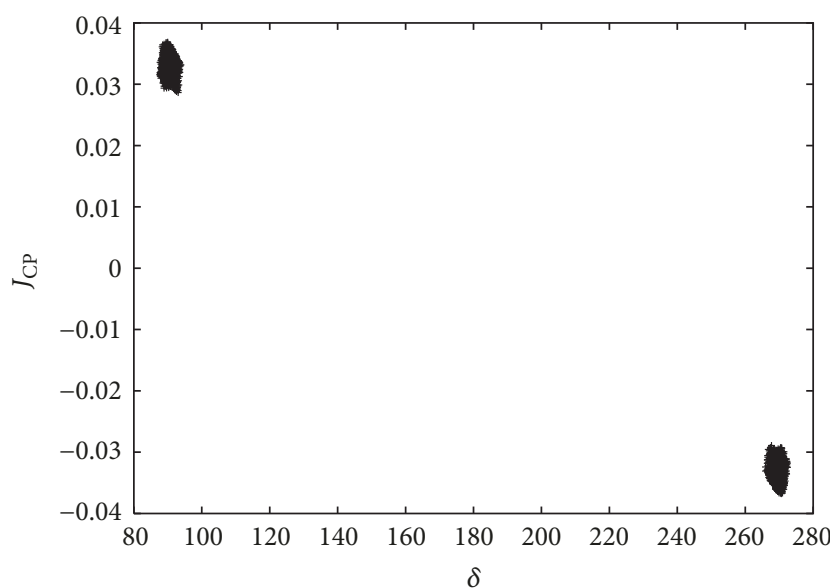

(d)

Figure 5: Case $T_{4}(\mathrm{NO})$ : scattering plots of Majorana phases, Dirac CP violating phase $(\delta)$, effective neutrino mass $|M|_{e e}$, and Jarlskog rephrasing invariant $\left(J_{\mathrm{CP}}\right)$ have been shown. All the phase angles $(\delta, \rho, \sigma)$ are measured in degrees and $|M|_{e e}$ is in $\mathrm{eV}$ unit.

Using the best fits from latest global neutrino oscillation data, the neutrino mass spectrum can be given as follows:

$$
\begin{aligned}
& m_{3}=\sqrt{\frac{\delta m^{2}}{\left(\zeta^{2}-\xi^{2}\right)}} \approx 4.87 \times 10^{-2} \mathrm{eV}, \\
& m_{2}=m_{3} \zeta \approx 2.85 \times 10^{-2} \mathrm{eV}, \\
& m_{1}=m_{3} \xi \approx 2.71 \times 10^{-2} \mathrm{eV},
\end{aligned}
$$

indicating that only NO is allowed. Since $T_{4}$ and $T_{5}$ are related due to permutation symmetry, therefore their phenomenological implications are similar. The phenomenological results for case $T_{5}$ can be derived from case $T_{4}$ using (3). The correlation plots for $\rho, \sigma, \delta,|M|_{e e}, J_{\mathrm{CP}}$ have been complied in Figure 6.
In the leading order of $s_{13}$ term, the effective mass term in $0 \nu \beta \beta$ decay can be approximated as

$$
|M|_{e e} \approx \frac{m_{3}}{2} \approx 2.43 \times 10^{-2} \mathrm{eV}
$$

which lies within the sensitivity limits of future $0 v \beta \beta$ decay experiments.

3.6. Case $T_{6}$. With the help of (6) and (9), we deduce the following analytical expressions in the leading order of $s_{13}$ term

$$
\begin{aligned}
& \frac{\lambda_{1}}{\lambda_{3}} \approx-\sec 2 \theta_{12}\left(c_{12}^{2}+e^{2 i \delta}\right) e^{i \delta}, \\
& \frac{\lambda_{2}}{\lambda_{3}} \approx \sec 2 \theta_{12}\left(s_{12}^{2}+e^{2 i \delta}\right) e^{i \delta} .
\end{aligned}
$$




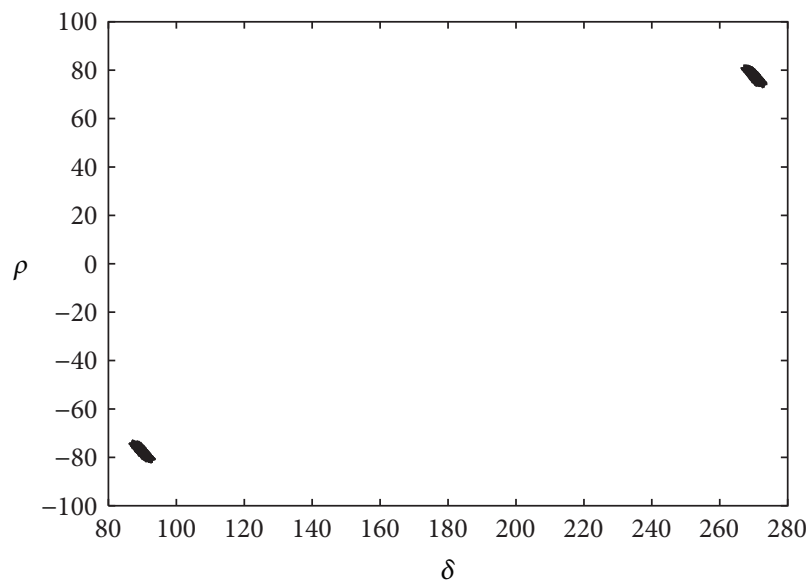

(a)

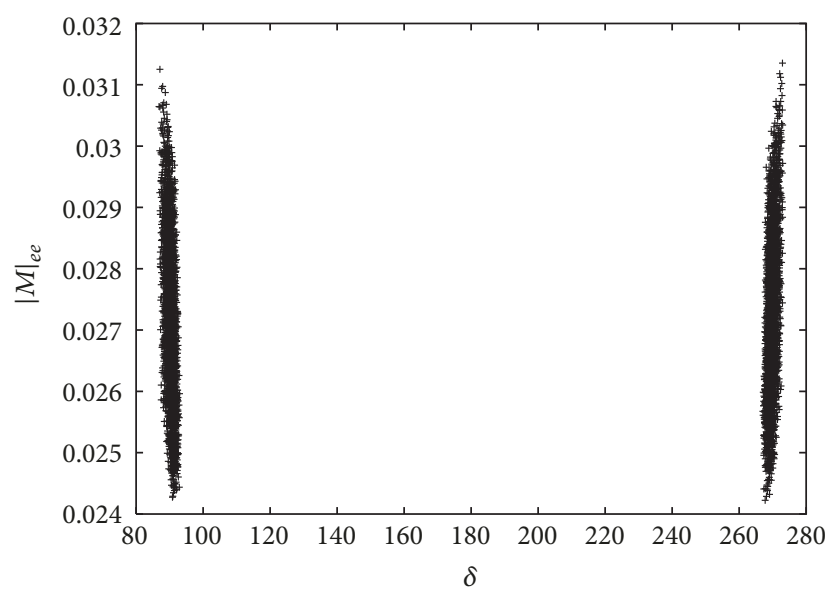

(c)

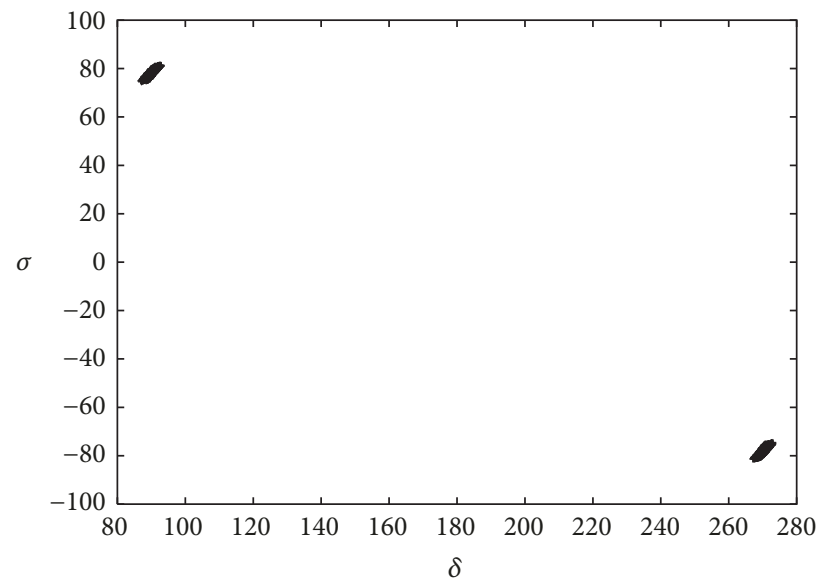

(b)

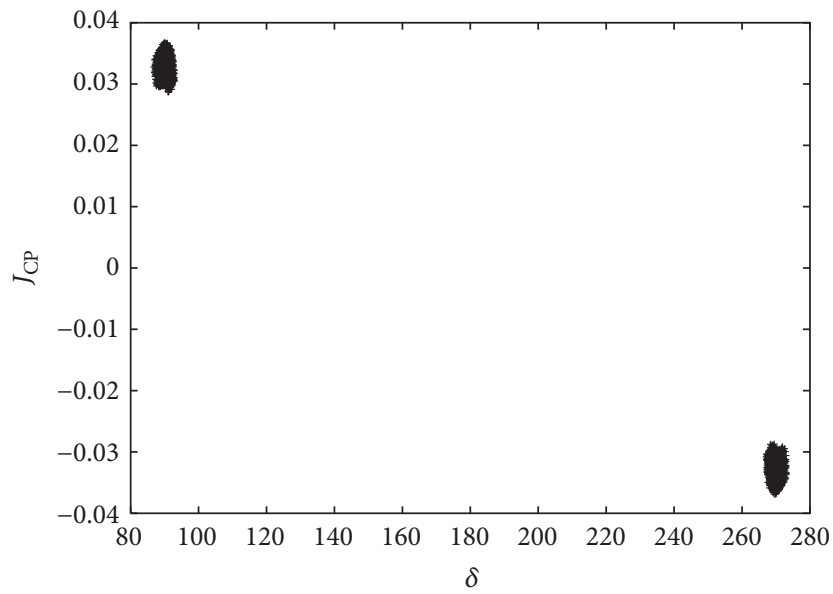

(d)

Figure 6: Case $T_{5}$ (NO): scattering plots of Majorana phases, Dirac CP violating phase $(\delta)$, effective neutrino mass $|M|_{e e}$, and Jarlskog rephrasing invariant $\left(J_{\mathrm{CP}}\right)$ have been shown. All the phase angles $(\delta, \rho, \sigma)$ are measured in degrees and $|M|_{e e}$ is in $\mathrm{eV}$ unit.

Using (38), we obtain the approximate relations for neutrino mass ratios

$$
\begin{aligned}
& \xi \approx \sec 2 \theta_{12} \sqrt{c_{12}^{4}+2 c_{12}^{2} \cos 2 \delta+1}, \\
& \zeta \approx \sec 2 \theta_{12} \sqrt{s_{12}^{4}+2 s_{12}^{2} \cos 2 \delta+1}
\end{aligned}
$$

and the Majorana CP violating phases

$$
\begin{aligned}
& \rho \approx \frac{1}{2} \tan ^{-1}\left(\frac{\sin \delta\left(\cos 2 \delta+c_{12}^{2}\right)+\sin 2 \delta \cos \delta}{\cos \delta\left(\cos 2 \delta+c_{12}^{2}\right)-\sin 2 \delta \sin \delta}\right) \\
& +O\left(s_{13}^{2}\right)
\end{aligned}
$$

$$
\begin{aligned}
\sigma \approx & \frac{1}{2} \tan ^{-1}\left(\frac{\sin \delta\left(\cos 2 \delta+s_{12}^{2}\right)+\sin 2 \delta \cos \delta}{\cos \delta\left(\cos 2 \delta+s_{12}^{2}\right)-\sin 2 \delta \sin \delta}\right) \\
& +O\left(s_{13}^{2}\right) .
\end{aligned}
$$

The correlation plots for $\rho, \sigma, \delta,|M|_{e e}, J_{\mathrm{CP}}$ have been complied in Figures 7(a), 7(b), 7(c), and 7(d). In Figures 7(a) and $7(\mathrm{~b})$ the parameter space of $\delta$ is found to be confined to very small regions. Also the Majorana phases $\rho$ and $\sigma$ also get restricted to $-54.7--44.7^{\circ} \oplus 45.3-54.5$. However, $J_{\mathrm{CP}}=0$ as evident in Figure $7(\mathrm{~d})$, which implies case $T_{6}$ points out the $\mathrm{CP}$ conservation.

From the analysis, out of six cases, only $T_{1}$ is found to be inconsistent with the experimental data for both normal and inverted mass ordering. For remaining cases the parameter space of CP violting phases $(\delta, \sigma, \rho)$, effective mass term $|M|_{e e}$, and neutrino masses $\left(m_{1}, m_{2}, m_{3}\right)$ is found to be 
TABLE 3: The allowed ranges of Dirac-like CP violating phase $\delta$, the Majorana phases $\rho, \sigma$, and three neutrino masses $m_{1}, m_{2}, m_{3}$ for the experimentally viable cases at $3 \sigma \mathrm{CL}$. Masses are in $\mathrm{eV}$.

\begin{tabular}{|c|c|c|c|c|}
\hline \multirow{3}{*}{$\begin{array}{l}\text { Cases } \\
T_{1}\end{array}$} & \multicolumn{2}{|c|}{ Normal mass ordering (NO) } & \multicolumn{2}{|c|}{ Inverted mass ordering (IO) } \\
\hline & $x$ & $x$ & $x$ & $x$ \\
\hline & $x$ & $x$ & $x$ & $x$ \\
\hline & $\times$ & $\times$ & $x$ & $\times$ \\
\hline \multirow{7}{*}{$T_{2}$} & $\delta=153.7^{\circ}-158.4^{\circ} \bigoplus 204.1^{\circ}-206.3^{\circ}$ & $m_{1}=0.0680-0.311$ & $\delta=0^{\circ}-21.06^{\circ} \bigoplus 126^{\circ}-156^{\circ}$ & $m_{1}=0.0425-0.357$ \\
\hline & $\rho=-64.9^{\circ}--58.5^{\circ} \oplus 58.6^{\circ}-64.3^{\circ}$ & $m_{2}=0.0712-0.314$ & $\oplus$ & $m_{2}=0.0435-0.359$ \\
\hline & $\sigma=-64.5^{\circ}--58.89^{\circ} \bigoplus 57.8^{\circ}-64.3^{\circ}$ & $m_{3}=0.0842-0.343$ & $204^{\circ}-233^{\circ}$ & $m_{3}=0.00098-0.359$ \\
\hline & & & $\oplus$ & \\
\hline & & & $340^{\circ}-360^{\circ}$ & \\
\hline & & & $\rho=-60^{\circ}-60^{\circ}$ & \\
\hline & & & $\sigma=-90^{\circ}--48.1^{\circ} \oplus 48.2^{\circ}-90^{\circ}$ & \\
\hline \multirow{7}{*}{$T_{3}$} & $\delta=12.49^{\circ}-25.9^{\circ} \bigoplus 334.9^{\circ}-348.8^{\circ}$ & $m_{1}=0.0291-0.690$ & $\delta=23.7^{\circ}-50.48^{\circ}$ & $m_{1}=0.0422-0.377$ \\
\hline & $\rho=-71.49^{\circ}--60.3^{\circ} \bigoplus 59.36^{\circ}-69.7^{\circ}$ & $m_{2}=0.0307-0.690$ & $\oplus$ & $m_{2}=0.0435-0.377$ \\
\hline & $\sigma=-73.34^{\circ}--59.62^{\circ} \bigoplus 57.87^{\circ}-72.7^{\circ}$ & $m_{3}=0.0480-0.690$ & $159.6^{\circ}-201.2^{\circ}$ & $m_{3}=0.00095-0.377$ \\
\hline & & & $\bigoplus$ & \\
\hline & & & $309.2^{\circ}-338.1^{\circ}$ & \\
\hline & & & $\rho=-60^{\circ}-60^{\circ}$ & \\
\hline & & & $\sigma=-90^{\circ}--48^{\circ} \bigoplus 48^{\circ}-90^{\circ}$ & \\
\hline \multirow{3}{*}{$T_{4}$} & $\delta=86.68^{\circ}-94.95^{\circ} \bigoplus 264.8^{\circ}-273.3^{\circ}$ & $m_{1}=0.0269-0.0406$ & $\times$ & $\times$ \\
\hline & $\rho=-82.87^{\circ}--1.29^{\circ} \bigoplus 69.57^{\circ}-82.08^{\circ}$ & $m_{2}=0.0280-0.0417$ & $\times$ & $\times$ \\
\hline & $\sigma=-82.87^{\circ}--71.7^{\circ} \bigoplus 70.5^{\circ}-82.08^{\circ}$ & $m_{3}=0.0516-0.0682$ & $x$ & $x$ \\
\hline \multirow{3}{*}{$T_{5}$} & $\begin{array}{c}\delta= \\
85.99^{\circ}-94.48^{\circ} \bigoplus^{2} 266.45^{\circ}-274.49^{\circ}\end{array}$ & $m_{1}=0.0269-0.0406$ & $x$ & $\times$ \\
\hline & 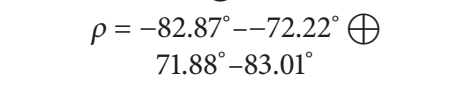 & $m_{2}=0.0280-0.0417$ & $\times$ & $x$ \\
\hline & $\sigma=-82.87^{\circ}--72.22^{\circ} \bigoplus 71.88-83.01^{\circ}$ & $m_{3}=0.0516-0.0682$ & $\times$ & $\times$ \\
\hline \multirow{9}{*}{$T_{6}$} & $\times$ & $\times$ & $\delta=53.25^{\circ}-68.5^{\circ}$ & $m_{1}=0.0449-0.0664$ \\
\hline & $x$ & $x$ & $\oplus$ & $m_{2}=0.0456-0.0672$ \\
\hline & $x$ & $x$ & $110.2^{\circ}-125^{\circ}$ & $m_{3}=0.0099-0.0400$ \\
\hline & $x$ & $\times$ & $\bigoplus$ & \\
\hline & $x$ & $x$ & $235.7^{\circ}-250^{\circ}$ & \\
\hline & $x$ & $x$ & $\oplus$ & \\
\hline & $x$ & $x$ & $291.3^{\circ}-309^{\circ}$ & \\
\hline & & & $\begin{array}{c}\rho=-54.73^{\circ}--44.7^{\circ} \\
45.36^{\circ}-54.5 \circ\end{array}$ & \\
\hline & & & 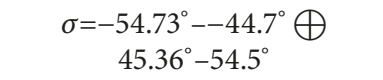 & \\
\hline
\end{tabular}

constrained to an appreciable extent at $3 \sigma$ CL. The allowed ranges of all the five viable cases for Dirac CP violating phase $(\delta)$, Majorana phases $(\rho, \sigma)$, and neutrino masses $\left(m_{1}, m_{2}, m_{3}\right)$ have been summarized in Table 3.

\section{Summary and Conclusion}

In the present work, we have systematically analyzed the texture one-zero Majorana mass matrix along with zero trace condition. In our analysis, we find that case $T_{1}$ with vanishing $(1,1)$ element of $M_{v}$ is ruled out with current experimental data. Therefore, out of six possible cases of one-zero texture with zero trace, only five, namely, $T_{2}, T_{3}, T_{4}, T_{5}$, and $T_{6}$, can survive the current experimental tests at $3 \sigma \mathrm{CL}$. The ongoing and future neutrino based experiments including neutrinoless double beta decay and cosmological experiments would test the validity of present texture zero analysis.

\section{Conflicts of Interest}

The authors declare that there are no conflicts of interest regarding the publication of this paper. 


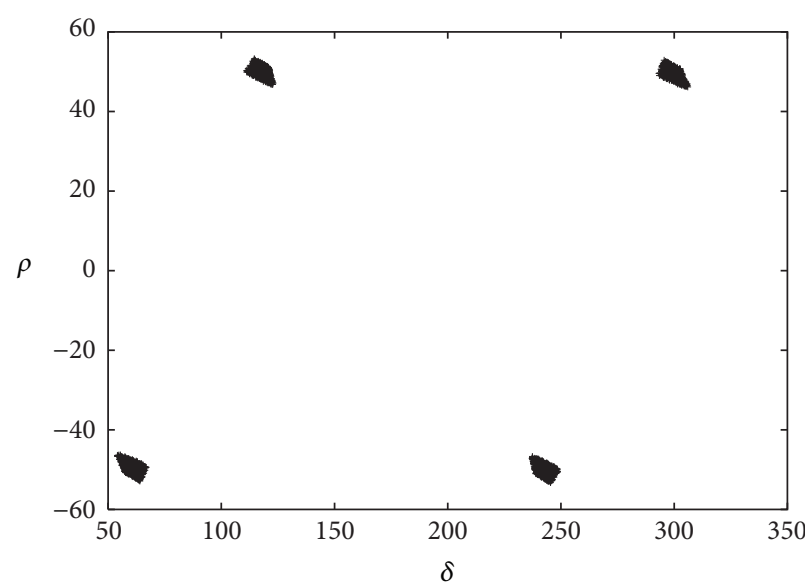

(a)

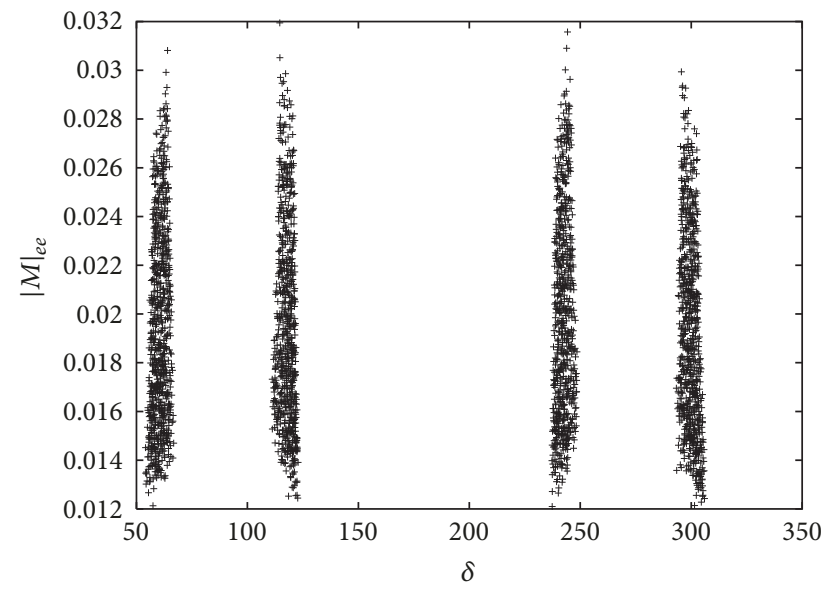

(c)

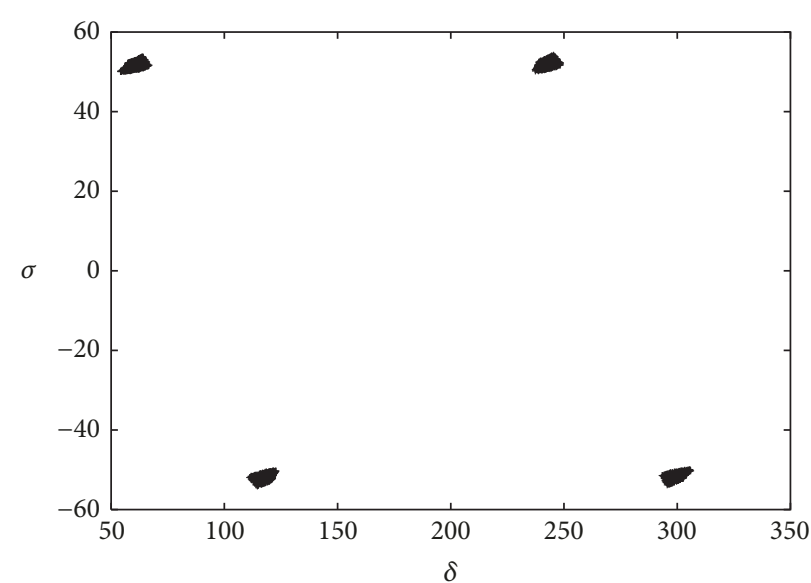

(b)

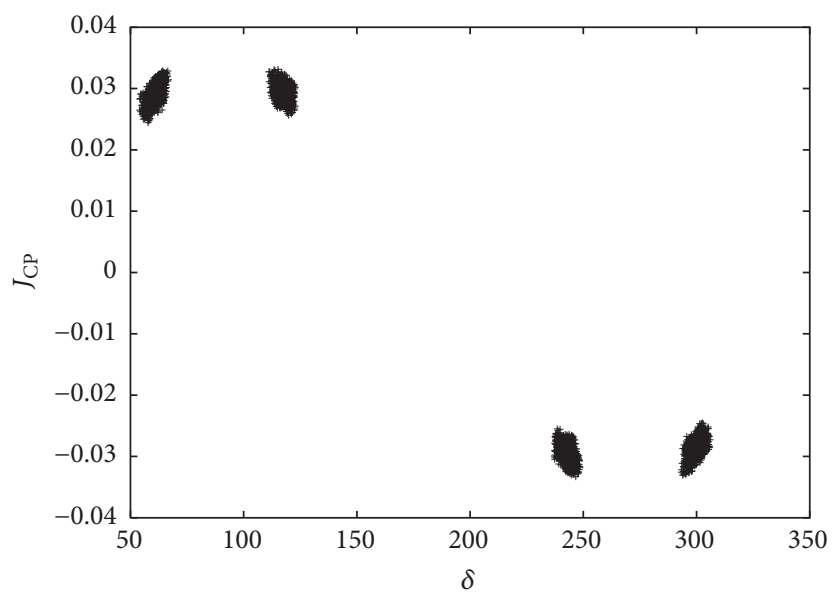

(d)

Figure 7: Case $T_{6}$ (IO): scattering plots of Majorana phases, Dirac CP violating phase $(\delta)$, effective neutrino mass $|M|_{e e}$, and Jarlskog rephrasing invariant $\left(J_{\mathrm{CP}}\right)$ have been shown. All the phase angles $(\delta, \rho, \sigma)$ are measured in degrees and $|M|_{e e}$ is in $\mathrm{eV}$ unit.

\section{Acknowledgments}

The author would like to thank the Director of National Institute of Technology Kurukshetra, for providing the necessary facilities to work.

\section{References}

[1] Y. Abe, T. Akiri, J. Dos Anjos et al., "Indication of reactor $\bar{v}_{e}$ disappearance in the double chooz experiment," Physical Review Letters, vol. 108, no. 13, 2012.

[2] F. P. An, J. Z. Bai, A. B. Balantekin et al., "Observation of electron-antineutrino disappearance at daya bay," Physical Review Letters, vol. 108, no. 17, 2012.

[3] S.-B. Kim and RENO collaboration, "Observation of reactor electron antineutrinos disappearance in the RENO experiment," Physical Review Letters, vol. 108, p. 191802, 2012.

[4] P. H. Frampton, S. L. Glashow, and D. Marfatia, "Zeroes of the neutrino mass matrix," Physics Letters B, vol. 536, no. 1-2, pp. 79-82, 2002.

[5] Z.-Z. Xing, "Texture zeros and majorana phases of the neutrino mass matrix," Physics Letters B, vol. 530, no. 1-4, pp. 159-166, 2002.
[6] R. Bipin, R. Desai, D. P. Roy, R. Alexander, and R. Vaucher, "Three-neutrino mass matrices with two texture zeros," Modern Physics Letters A, vol. 18, no. 20, pp. 1355-1365, 2003.

[7] A. Merle and W. Rodejohann, "Elements of the neutrino mass matrix: allowed ranges and implications of texture zeros," Physical Review D, vol. 73, no. 7, Article ID 073012, 11 pages, 2006.

[8] S. Dev, S. Kumar, S. Verma, and S. Gupta, "Phenomenological implications of a class of neutrino mass matrices," Nuclear Physics B, vol. 784, no. 1-2, pp. 103-117, 2007.

[9] S. Dev, S. Kumar, S. Verma, and S. Gupta, "Phenomenology of two-texture zero neutrino mass matrices," Physical Review D, vol. 76, no. 1, Article ID 013002, 15 pages, 2007.

[10] M. Randhawa, G. Ahuja, and M. Gupta, "Implications of Fritzsch-like lepton mass matrices," Physics Letters B, vol. 643, no. 3-4, pp. 175-181, 2006.

[11] G. Ahuja, S. Kumar, M. Randhawa, M. Gupta, and S. Dev, "Texture 4 zero Fritzsch-like lepton mass matrices," Physical Review D, vol. 76, no. 1, Article ID 013006, 2007.

[12] S. Kumar, "Implications of a class of neutrino mass matrices with texture zeros for nonzero $\theta_{13}$," Physical Review D, vol. 84, no. 7, Article ID 077301, 2011. 
[13] P. O. Ludl, S. Morisi, and E. Peinado, "The reactor mixing angle and CP violation with two texture zeros in the light of T2K," Nuclear Physics B, vol. 857, no. 3, pp. 411-423, 2012.

[14] H. Itoyama and N. Maru, "Flavor mixings and textures of the fermion mass matrices," International Journal of Modern Physics A, vol. 27, no. 31, Article ID 1250159, 2012.

[15] D. Meloni and G. Blankenburg, "Fine-tuning and naturalness issues in the two-zero neutrino mass textures," Nuclear Physics $B$, vol. 867, no. 3, pp. 749-762, 2013.

[16] W. Grimus and J. Ludl, "Two-parameter neutrino mass matrices with two texture zeros," Journal of Physics G: Nuclear and Particle Physics, vol. 40, Article ID 125003, p. 055003, 2013.

[17] S. Sharma, P. Fakay, G. Ahuja, and M. Gupta, "Majorana neutrinos and non minimal lepton mass textures," https://arxiv.org/abs/1402.1598.

[18] P. O. Ludl and W. Grimus, "A complete survey of texture zeros in the lepton mass matrices," Journal of High Energy Physics, vol. 2014, 2014.

[19] H. Fritzsch, Z. Z. Xing, and S. Zhou, "Two-zero textures of the Majorana neutrino mass matrix and current experimental tests," Journal of High Energy Physics, vol. 2011, no. 09, p. 083, 2011.

[20] E. I. Lashin and N. Chamoun, "One-zero textures of Majorana neutrino mass matrix and current experimental tests," Physical Review D, vol. 85, no. 11, p. 113011, 2012.

[21] K. N. Deepthi, S. Gollu, and R. Mohanta, "Neutrino mixing matrices with relatively large $\theta 13$ and with texture one-zero," The European Physical Journal C, vol. 72, no. 2, pp. 1-18, 2012.

[22] J. Liao, D. Marfatia, and K. Whisnant, "Seesaw mechanism with four texture zeros in the neutrino Yukawa matrix," Physical Review D: Particles, Fields, Gravitation and Cosmology, vol. 87, no. 7, p. 073013, 2013.

[23] J. Liao, D. Marfatia, and K. Whisnant, "One diagonal texture or cofactor zero of the neutrino mass matrix," Physical Review D, vol. 88, no. 3, p. 033011, 2013.

[24] H. Fritzsch and Z. Z. Xing, "Mass and flavor mixing schemes of quarks and leptons," Progress in Particle and Nuclear Physics, vol. 45, no. 1, 2000, arXiv: hep-ph/9912358.

[25] Z. Z. Xing, "Texture zeros and CP-violating phases in the neutrino mass matrix," arXiv: hep-ph/0406049.

[26] M. Singh, G. Ahuja, and M. Gupta, "Revisiting the texture zero neutrino mass matrices," PTEP, vol. 2016, no. 8, p. 123, 2016.

[27] X. Sun and W. Liu, "Improved black hole entropy calculation without cutoff," Modern Physics Letters A, vol. 19, no. 9, 2004.

[28] G. C. Branco, R. González Felipe, F. R. Joaquim, and T. Yanagida, "Removing ambiguities in the neutrino mass matrix," Physics Letters B, vol. 562, no. 3-4, pp. 265-272, 2003.

[29] Z. Z. Xing, "The majorana neutrino mass matrix with one texture zero and one vanishing eigenvalue," Physical Review D, vol. 69, p. 013006, 2004, arXiv: hep-ph/0307007.

[30] W. Rodejohann, M. Tanimoto, and A. Watanabe, "Relating large $U_{\mathrm{e} 3}$ to the ratio of neutrino mass-squared differences," Physics Letters B, vol. 710, no. 4-5, 2012.

[31] L. Lavoura, W. Rodejohann, and A. Watanabe, "Reproducing lepton mixing in a texture zero model," Physics Letters B, vol. 726, no. 1-3, 2013.

[32] R. R. Gautam, M. Singh, and M. Gupta, "Neutrino mass matrices with one texture zero and a vanishing neutrino mass," Physical Review D, vol. 92, no. 1, Article ID 024001, p. 013006, 2015.
[33] D. Black, A. H. Fariborz, S. Nasri, and J. Schechter, "Complementary ansatz for the neutrino mass matrix," Physical Review $D$, vol. 62, p. 073015, 2000, arXiv: hep-ph/0004105.

[34] X. G. He and A. Zee, "Neutrino masses with "zero sum" condition: $m_{v 1}+m_{\nu 2}+m_{\nu 3}=0$," Physical Review D, vol. 68, p. 037302, 2003, arXiv: hep-ph/0302201.

[35] A. Zee, "A theory of lepton number violation and neutrino Majorana masses," Physics Letters B, vol. 93, no. 4, pp. 389-393, 1980.

[36] H. A. Alhendi, E. I. Lashin, and A. A. Mudlej, "Textures with two traceless submatrices of the neutrino mass matrix," Physical Review D, vol. 77, no. 1, p. 013009, 2008.

[37] W. Rodejohann, "Neutrino mass matrices leaving no trace," Physics Letters B, vol. 579, no. 1-2, pp. 127-139, 2004.

[38] G. L. Fogli, E. Lisi, A. Marrone et al., "Evidence of $\theta_{13}>0$ from global neutrino data analysis," Physical Review D, vol. 84, no. 5, 2011.

[39] D. V. Forero, M. Tórtola, and J. W. F. Valle, "Neutrino oscillations refitted," Physical Review D, vol. 90, no. 9, p. 093006, 2014.

[40] F. T. Avignone III, S. R. Elliott, and J. Engel, "Double beta decay, Majorana neutrinos, and neutrino mass," Reviews of Modern Physics, vol. 80, no. 2, p. 481, 2008.

[41] J. J. Gomez-Cadenas, J. Martin-Albo, M. Mezzetto, F. Monrabal, and M. Sorel, "The search for neutrinoless double beta decay," Italian Physical Science, no. 2, 2012.

[42] S. M. Bilenky and C. Giunti, "Neutrinoless double-beta decay: a brief review," Modern Physics Letters A, vol. 27, no. 13, Article ID 1230015, 2012.

[43] W. Rodejohann, "Neutrino-less double beta decay and particle physics," International Journal of Modern Physics E, vol. 20, no. 9, p. 1833, 2011.

[44] C. Arnaboldi et al., "First results on neutrinoless double beta decay of 130 Te with the calorimetric CUORICINO experiment," Physics letters B, vol. 584, p. 260, 2004.

[45] C. Arnaboldi et al., "CUORE: a cryogenic underground observatory for rare events," Nuclear Instruments and Methods in Physics Research A, vol. 518, p. 775.

[46] I. Abt et al., "A new 76ge double beta decay experiment at LNGS," [GERDA Collaboration] hep-ex/0404039.

[47] R. Gaitskell et al., "A new evaluation of the pion weak form factors," [Majorana Collaboration] nucl-ex/0311013.

[48] A. S. Barabash, "Average (recommended) half-life values for two neutrino double beta decay," Czechoslovak Journal of Physics, vol. 52, p. 567, 2002.

[49] M. Danilov, R. DeVoec, A. Dolgolenkod et al., "Detection of very small neutrino masses in double-beta decay using laser tagging," Physics Letters B, vol. 480, no. 1-2, pp. 12-18, 2000.

[50] H. V. Klaapdor-Kleingrothaus, A. Dietz, G. Baudis et al., "Latest results from the HEIDELBERG-MOSCOW double beta decay experiment," The European Physical Journal A - Hadrons and Nuclei, vol. 12, no. 2, pp. 147-154, 2001.

[51] P. A. R. Ade, N. Aghanim, C. Armitage-Caplan et al., "Planck 2013 results. XVI. cosmological parameters," Astronomye Astrophysics: World Wide Astronomical and Astrophysical Research, vol. 571, 2014. 

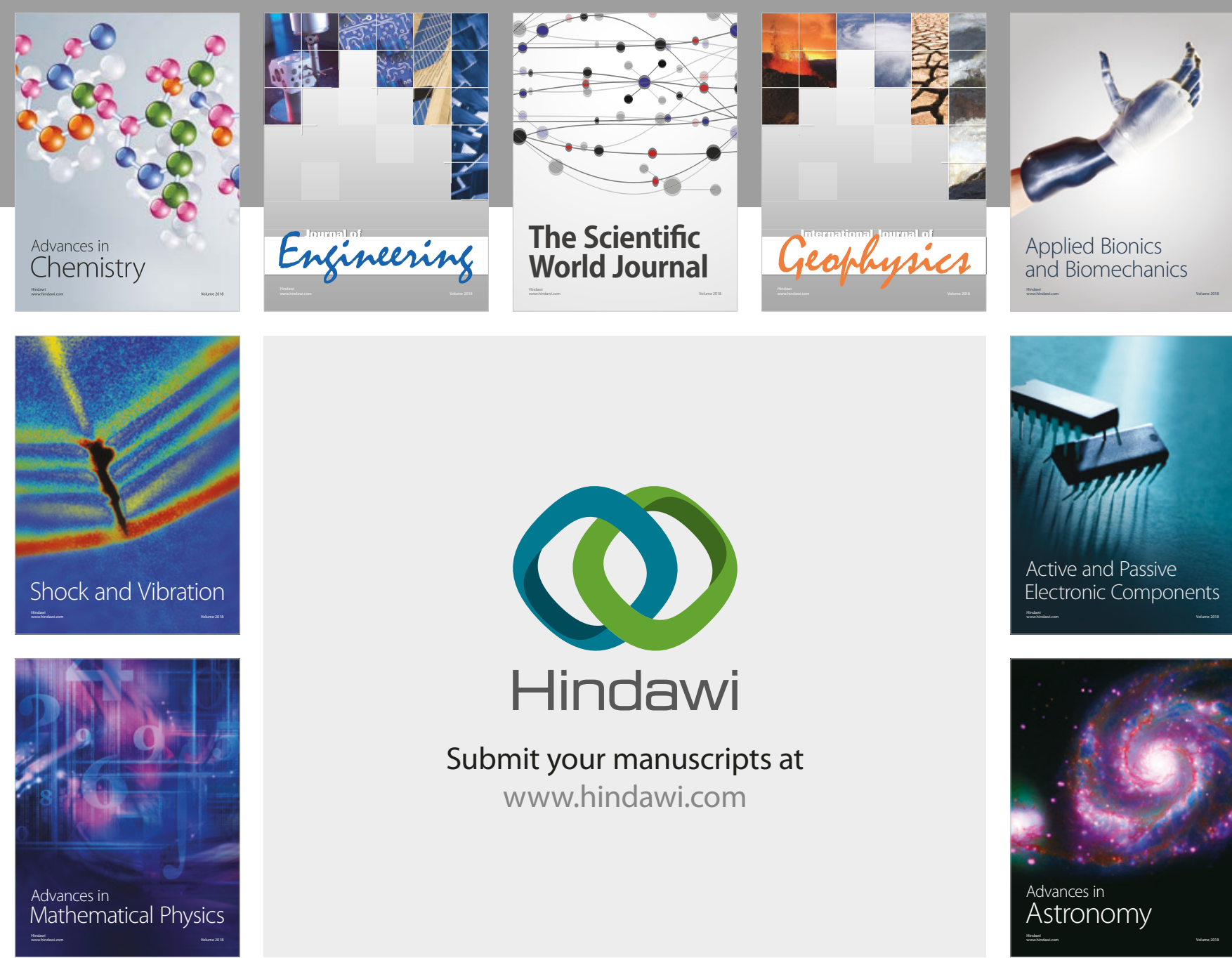

Submit your manuscripts at

www.hindawi.com

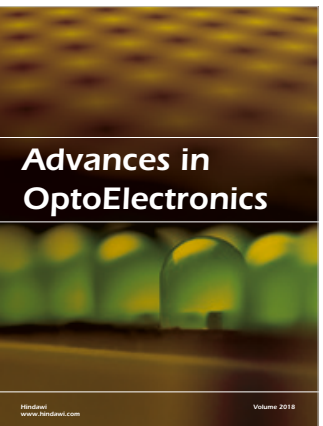

\section{Rotcting Machinery}
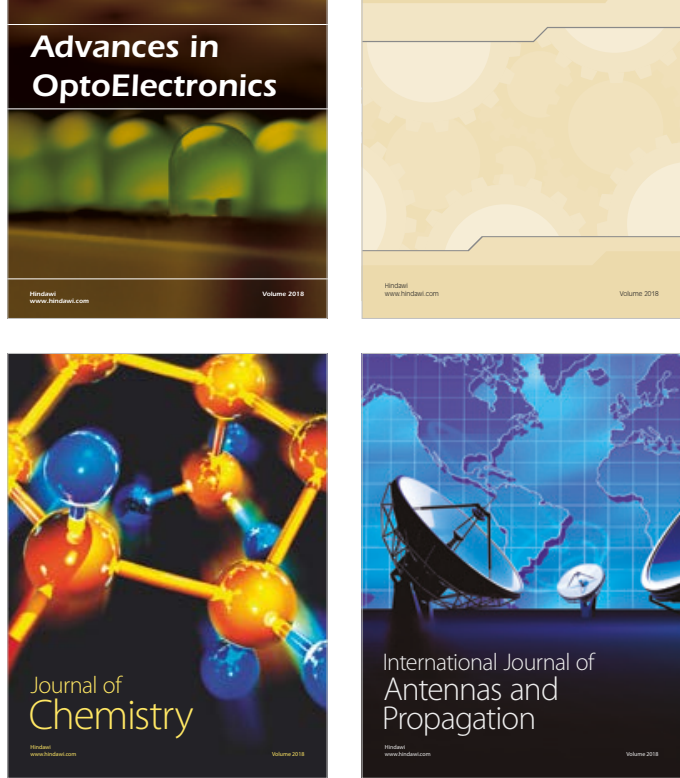

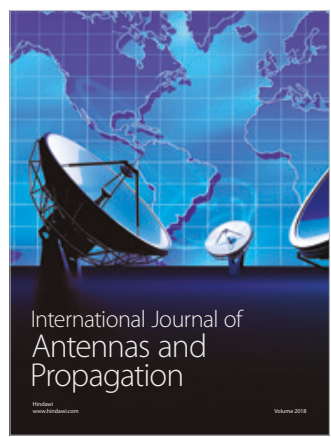

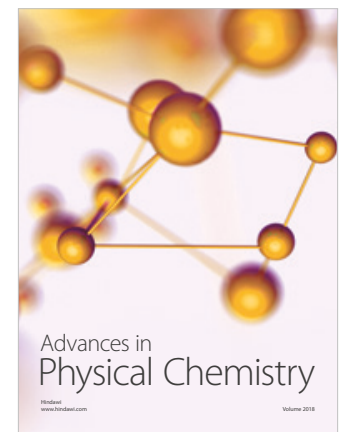

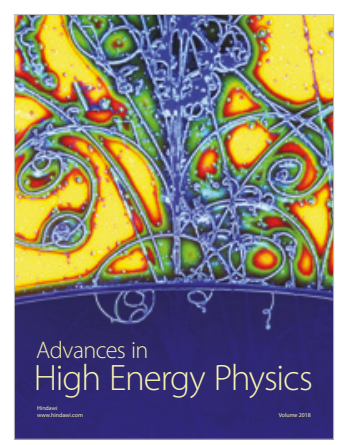

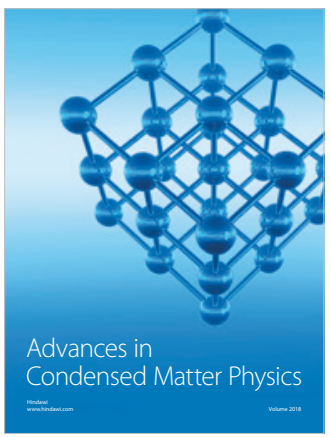

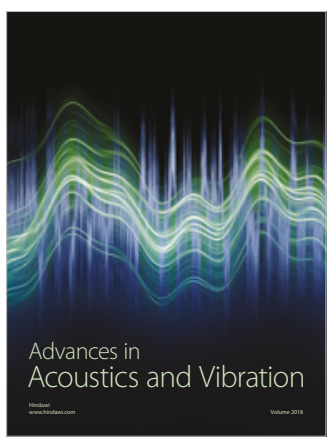

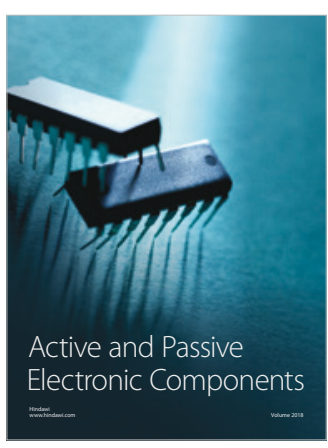
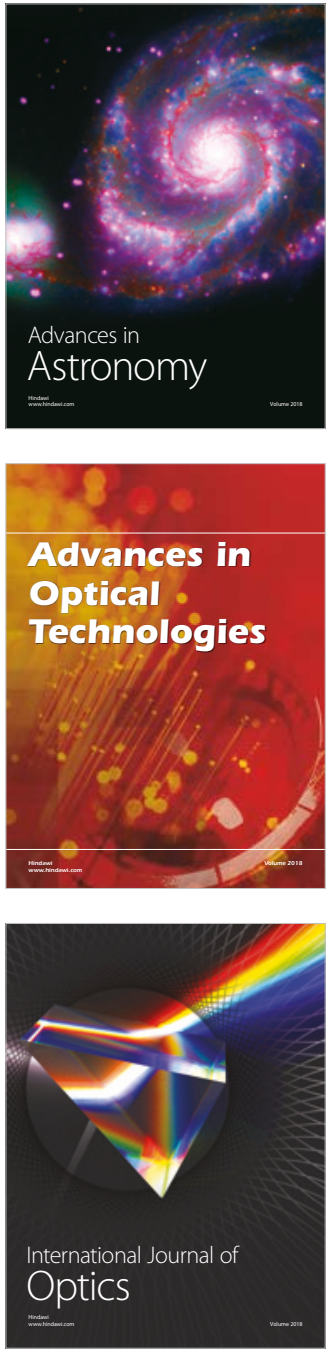